\title{
Invertibility and weak continuity of the determinant for the modelling of cavitation and fracture in nonlinear elasticity
}

\author{
Duvan Henao • Carlos Mora-Corral
}

21 September, 2009

\begin{abstract}
In this paper we present and analyze a variational model in nonlinear elasticity that allows for cavitation and fracture. The main idea to unify the theories of cavitation and fracture is to regard both cavities and cracks as phenomena of creation of new surface. Accordingly, we define a functional that measures the area of the created surface. This functional has relationships with the theory of Cartesian currents. We show that the boundedness of that functional implies the sequential weak continuity of the determinant of the deformation gradient, and that the weak limit of one-to-one a.e. deformations is also one-to-one a.e. We then use these results to obtain existence of minimizers of variational models that incorporate the elastic energy and this created surface energy, taking into account the orientation-preserving and the non-interpenetration conditions.
\end{abstract}

Keywords Cavitation · Fracture · Nonlinear elasticity · Surface energy · Variational models

Mathematics Subject Classification (2000) 49Q20 - 49J45 - 74G65 - 74B20 •

$28 \mathrm{~A} 75 \cdot 74 \mathrm{R} 10$

\section{Introduction}

The fundamental problem in elastostatics is to ascertain whether the elastic energy

$$
\int_{\Omega} W(\mathbf{x}, D \mathbf{u}(\mathbf{x})) \mathrm{d} \mathbf{x}
$$

D. Henao

Laboratoire Jacques-Louis Lions, Université Pierre et Marie Curie, Boîte Courrier 187, 75252 Paris Cedex 05, France.

Tel.: +33 (0) 144277203

E-mail: henao@ann.jussieu.fr

C. Mora-Corral

Basque Center for Applied Mathematics (BCAM), Parque Tecnológico de Bizkaia, Edif. 500, E-48160 Derio (Vizcaya), Spain.

Tel.: +34946567842

E-mail: mora@bcamath.org 
of a deformation $\mathbf{u}: \Omega \rightarrow \mathbb{R}^{3}$ has a minimum in a suitable class of deformations satisfying certain invertibility and boundary conditions. Here $\Omega$ is an open bounded subset of $\mathbb{R}^{3}$ representing the reference configuration of the body, and $W: \Omega \times \mathbb{R}^{3 \times 3} \rightarrow$ $\mathbb{R} \cup\{\infty\}$ is the elastic stored-energy function of the material. The usual approach for finding such minimizers is the direct method of the calculus of variations. As shown in the pioneering paper of Ball [6], the sequential weak continuity of the determinant of the deformation gradient $D \mathbf{u}$ is an important ingredient to make the direct method work. This continuity property states that if a sequence $\mathbf{u}_{j}$ of deformations weakly converges to $\mathbf{u}$ in some Sobolev space, and some further conditions hold, then the sequence $\operatorname{det} D \mathbf{u}_{j}$ weakly converges to $\operatorname{det} D \mathbf{u}$ in $L^{1}$.

Ball's result and many of its refinements (e.g., $[10,32,27,33,35])$ are unable to deal with the phenomenon of cavitation, since in their functional setting any deformation that exhibit cavitation has infinite elastic energy. This is due to the strong coercivity assumptions on the stored-energy function $W$. To illustrate this, we mention that in Müller, Tang and Yan [35] the existence of minimizers is proved under the coercivity assumption

$$
W(\mathbf{x}, \mathbf{F}) \geq c_{1}|\mathbf{F}|^{2}+c_{1}|\operatorname{cof} \mathbf{F}|^{\frac{3}{2}}-c_{2}, \quad \mathbf{x} \in \Omega, \quad \mathbf{F} \in \mathbb{R}^{3 \times 3},
$$

for some $c_{1}, c_{2}>0$. These growth conditions are incompatible with the radial deformation $\mathbf{u}: B(\mathbf{0}, 1) \rightarrow \mathbb{R}^{3}$ defined as $\mathbf{u}(\mathbf{x}):=r(|\mathbf{x}|) \frac{\mathbf{x}}{\mathbf{x} \mid}$, which produces a cavity at the origin of radius $r(0)$. Here, $r:[0, \infty) \rightarrow(0, \infty)$ is a given $C^{1}$ function. Indeed, it is immediate to check that this $\mathbf{u}$ is in $W^{1, p}$ for all $p<3$, whereas cof $D \mathbf{u}$ is in $L^{q}$ for all $q<\frac{3}{2}$, but fails to belong to $L^{\frac{3}{2}}$. In fact, Ball and Murat [10] showed that if cavitation is energetically favourable, then the weak continuity of the determinant fails, and so does the weak lower semicontinuity of the energy (1). Hence the direct method of the calculus of variations is inapplicable.

The first mathematical theory for cavitation in the context of nonlinear elasticity is due to Ball [8], where he worked in the restricted framework of radial cavitations. The full three-dimensional case was treated by Müller and Spector [34]. The starting point of their approach is that the total energy of a deformation must be the sum of the elastic energy and a surface energy due to the formation of cavities. Thus, they proposed to minimize the energy

$$
\int_{\Omega} W(\mathbf{x}, D \mathbf{u}(\mathbf{x})) \mathrm{d} \mathbf{x}+\operatorname{Per} \mathbf{u}(\Omega),
$$

where Per denotes the perimeter of a set. Despite some drawbacks, the term Per $\mathbf{u}(\Omega)$ measures somehow the area of the created cavities (together with the area of the image of $\partial \Omega$ ). They proved the existence of minimizers satisfying, in addition, some invertibility conditions.

The theory of brittle fracture, within the variational context of nonlinear elasticity, was proposed by Francfort and Marigo [22], and based on Griffith's classic theory. Fracture (like cavitation) is a genuinely time-dependent problem. There has been a huge development and consequent understanding of the theory of brittle fracture in the last decade, mainly in the quasistatic case (see, e.g., $[18,17,21,15,12,16]$ or the review paper [11]), but many fundamental problems remain unsolved. In short, the problem in the static case is to find minimizers of the energy

$$
\int_{\Omega} W(\mathbf{x}, \nabla \mathbf{u}(\mathbf{x})) \mathrm{d} \mathbf{x}+\mathcal{H}^{2}\left(J_{\mathbf{u}}\right)
$$


The novelty is the term $\mathcal{H}^{2}\left(J_{\mathbf{u}}\right)$, which measures the area of the set of jump discontinuities of $\mathbf{u}$, and accounts for the energy due to fracture. The deformation $\mathbf{u}$ is typically supposed to lie in the space $S B V$ or $G S B V$, so that the matrix $\nabla \mathbf{u}$ denotes the approximate differential of $\mathbf{u}$, and not its distributional derivative. The existence of minimizers for (3) has been proved in $S B V$ under the extra assumption of an $L^{\infty}$ a priori bound on the class of admissible deformations, or in $G S B V$ under $L^{1}$ coercivity of the energy (see $[2,23])$. In this paper, we start from the $S B V$ theory of static fracture, and try to conciliate this theory with that of cavitation.

Our desire to build a theory that encompasses cavitation and fracture was partly motivated by the experimental observations of Petrinic et al. $[37,38]$ on ductile fracture of titanium alloys. In their experiments, the material was subjected to a tensile stress, and they show that fracture is preceded by the growth and coalescence of voids. In addition, cavitation and fracture are also related in elastomers. In particular, the works of Williams and Schapery [42] and of Gent and Wang [25] (see also the review paper [24]) suggest that the strains at the cavity surface produced during cavitation are so large that fracture occurs at the same time.

If one wishes to propose a theory within the variational context of nonlinear elasticity that allows for both cavitation and fracture, one cannot just put the two theories together. Indeed, an important ingredient in the theory of cavitation of [34] is that the admissible deformations must satisfy condition (INV). This condition roughly expresses that cavities produced in one part of the body cannot be filled by material from elsewhere. It turns out that the mere formulation of condition (INV) requires some properties of continuity of the deformation; in loose terms, that the restriction of the deformation to almost every surface is continuous. This continuity property is satisfied if, for example, the deformation lies in $W^{1, p}$ for some $p>2$. In contrast, whenever fracture occurs, condition (INV) cannot even be formulated, since that continuity property is clearly false. Consequently, it seems that a previous step in the construction of a theory that allows for cavitation and fracture is the development of a theory of cavitation that makes no use of condition (INV). Since, as shown in [34], condition (INV) was essential to rule out some pathological examples, perhaps the first task to do is to define a surface energy, in the spirit of $\operatorname{Per} \mathbf{u}(\Omega)$, that measures the area of the created surface and is able to rule out those counterexamples.

Our idea to unify fracture and cavitation is to regard both as processes of creation of surface. For a deformation $\mathbf{u}: \Omega \rightarrow \mathbb{R}^{3}$, we will define the surface energy $\mathcal{E}(\mathbf{u})$ as the supremum, when $\mathbf{f} \in C_{c}^{\infty}\left(\Omega \times \mathbb{R}^{3}, \mathbb{R}^{3}\right)$ and $\|\mathbf{f}\|_{\infty} \leq 1$, of the quantity

$$
\int_{\Omega}\left[\operatorname{cof} \nabla \mathbf{u}(\mathbf{x}) \cdot D_{\mathbf{x}} \mathbf{f}(\mathbf{x}, \mathbf{u}(\mathbf{x}))+\operatorname{det} \nabla \mathbf{u}(\mathbf{x}) \operatorname{div}_{\mathbf{y}} \mathbf{f}(\mathbf{x}, \mathbf{u}(\mathbf{x}))\right] \mathrm{d} \mathbf{x} .
$$

It is not easy to motivate the definition of $\mathcal{E}$ as a surface energy in just a few lines, so we postpone this motivation until Sections 3 and 4 . In fact, the full proof that the functional $\mathcal{E}$ accounts for the area of the surface created by $\mathbf{u}$ will be given in a forthcoming paper [30]. For the moment, let us accept that $\mathcal{E}$ measures the area of the new surface created by the deformation $\mathbf{u}$. It makes sense, therefore, to propose a model based on the minimization of the energy

$$
\int_{\Omega} W(\mathbf{x}, \nabla \mathbf{u}(\mathbf{x})) \mathrm{d} \mathbf{x}+\mathcal{E}(\mathbf{u})
$$

Section 5 will show that this functional has indeed a minimum. The term $\mathcal{E}(\mathbf{u})$, moreover, is such that it is not necessary to use condition (INV) in order to obtain the 
existence of minimizers: the natural condition of invertibility a.e. (introduced by Ball [7]) can be used instead.

The main ingredient in the proof of the existence result mentioned above is the following theorem (see Theorems 2 and 3 for a stronger formulation), which states that both the weak continuty of the determinant and the stability of the property of invertibility a.e. under weak limits can be obtained as a consequence of the boundedness of the surface energy.

Theorem 1 For each $j \in \mathbb{N}$, let $\mathbf{u}_{j}, \mathbf{u}: \Omega \rightarrow \mathbb{R}^{3}$ be approximately differentiable in almost all $\Omega$. Suppose that there exists $\theta \in L^{1}(\Omega)$ such that $\theta>0$ a.e., and

$$
\begin{aligned}
& \mathbf{u}_{j} \rightarrow \mathbf{u} \text { a.e., } \quad \operatorname{cof} \nabla \mathbf{u}_{j} \rightarrow \operatorname{cof} \nabla \mathbf{u} \text { in } L^{1}\left(\Omega, \mathbb{R}^{3 \times 3}\right), \quad \operatorname{det} \nabla \mathbf{u}_{j} \rightarrow \theta \text { in } L^{1}(\Omega) \\
& \text { as } j \rightarrow \infty \text {. Assume that for each } j \in \mathbb{N} \text {, the function } \mathbf{u}_{j} \text { is one-to-one a.e., } \operatorname{det} \nabla \mathbf{u}_{j}>0 \\
& \text { a.e., and } \\
& \qquad \sup _{j \in \mathbb{N}} \mathcal{E}\left(\mathbf{u}_{j}\right)<\infty .
\end{aligned}
$$

Then $\mathbf{u}$ is one-to-one a.e., and $\theta=\operatorname{det} \nabla \mathbf{u}$ a.e.

With Theorem 1, the existence of minimizers of the energy functional (4) becomes then a standard task.

We finally mention that the functional $\mathcal{E}$ has some connections with the theory of Cartesian currents. This relationship will be explained in Section 4, but we also remark that no knowledge of the theory of currents is needed in this paper.

The plan of the paper is as follows. Section 2 introduces the general notation of the paper and recall some important preliminary results, such as the change of variables formula. Section 3 starts with a motivation of the definition of surface energy. We then define a tentative surface energy $\overline{\mathcal{E}}$ and study some of its properties, the most important one being that its boundedness guarantees that the limit of a sequence of one-to-one a.e. maps is also one-to-one a.e. In Section 4 we define the surface energy $\mathcal{E}$ mentioned above, which is a stronger energy than $\overline{\mathcal{E}}$. Then we show, using ideas of Müller [32] and of Giaquinta, Modica and Souček [28], that its boundedness implies a property on the weak continuity of the determinant. In Section 5 we use the result on the stability under the limit of the injectivity property proved in Section 3, together with the result on the continuity of the determinant proved in Section 4 , in order to prove the results on the existence of minimizers for our model of elastic solids that may undergo cavitation and fracture. In Section 6 we prove that $\overline{\mathcal{E}}$ and $\mathcal{E}$ do not coincide, and show by means of an example that $\overline{\mathcal{E}}$ does not correspond to a physical quantity. Finally, in Section 7 we make some comments on other models related to ours that have been previously proposed.

\section{Notation and preliminaries}

In this section we set the general notation of this paper, and state some important preliminary results.

We will work in dimension $n$, and tacitly assume that $n \geq 2$. Our body will be represented by a bounded open set $\Omega$ of $\mathbb{R}^{n}$. Sometimes, $\Omega$ is assumed to have a strongly Lipschitz boundary, so that the trace of a Sobolev function on the topological boundary $\partial \Omega$ of $\Omega$ is defined. The closure of $\Omega$ is denoted by $\bar{\Omega}$. 
Unless otherwise stated, expressions like measurable or a.e. refer to the Lebesgue measure in $\mathbb{R}^{n}$, which is denoted by $\mathcal{L}^{n}$. The $m$-dimensional Hausdorff measure will be indicated by $\mathcal{H}^{m}$. Usually, $m$ will be $n-1$.

Our basic object (the deformation) will be a measurable map $\mathbf{u}: \Omega \rightarrow \mathbb{R}^{n}$. Vectorvalued quantities will be written in boldface. Coordinates in the reference configuration will generically be denoted by $\mathbf{x}$, while coordinates in the deformed configuration by $\mathbf{y}$. The divergence operator in the reference configuration (so with respect to the $\mathbf{x}$ coordinates) is denoted by Div, while div is the divergence in the deformed configuration (with respect to $\mathbf{y}$ ).

The open ball of radius $r>0$ centred at $\mathbf{x} \in \mathbb{R}^{n}$ is denoted by $B(\mathbf{x}, r)$, whereas the closed ball is $\bar{B}(\mathbf{x}, r)$.

If $\mathbf{u}$ is a Sobolev function, $D \mathbf{u}$ denotes the distributional derivative of $\mathbf{u}$. Note that we do not identify functions that are equal almost everywhere.

We will say that $\mathbf{x}_{0} \in \Omega$ is a Lebesgue point of the measurable map $\mathbf{u}: \Omega \rightarrow \mathbb{R}^{n}$ when

$$
\lim _{r \searrow 0} f_{B\left(\mathbf{x}_{0}, r\right)}\left|\mathbf{u}(\mathbf{x})-\mathbf{u}\left(\mathbf{x}_{0}\right)\right| \mathrm{d} \mathbf{x}=0 .
$$

As usual, $f_{A}$ denotes $\frac{1}{\mathcal{L}^{n}(A)} \int_{A}$ for any measurable set $A$. We will say that $\mathbf{u}$ is approximately differentiable at $\mathbf{x}_{0} \in \Omega$ if there exists $\mathbf{L} \in \mathbb{R}^{n \times n}$ such that

$$
\lim _{r \searrow 0} f_{B\left(\mathbf{x}_{0}, r\right)}\left|\frac{\mathbf{u}(\mathbf{x})-\mathbf{u}\left(\mathbf{x}_{0}\right)-\mathbf{L}\left(\mathbf{x}-\mathbf{x}_{0}\right)}{r}\right| \mathrm{d} \mathbf{x}=0 .
$$

This $\mathbf{L}$ is uniquely determined, will be called the approximate differential of $\mathbf{u}$ at $\mathbf{x}_{0}$, and will be denoted by $\nabla \mathbf{u}\left(\mathbf{x}_{0}\right)$. Note that if $\mathbf{u}$ is approximately differentiable at $\mathbf{x}_{0}$, then $\mathbf{x}_{0}$ is a Lebesgue point of $\mathbf{u}$. We will say that a map $\mathbf{u}: \Omega \rightarrow \mathbb{R}^{n}$ is approximately differentiable in almost all $\Omega$ when it is measurable and approximately differentiable at almost each point of $\Omega$. It is worthwhile to recall that the Calderón-Zygmund theorem asserts that if $\mathbf{u}$ is a $B V_{\text {loc }}$ function, then it is approximately differentiable in almost all its domain, and $\nabla \mathbf{u}$ is the density of the absolutely continuous part of the measure $D \mathbf{u}$. In particular, if $\mathbf{u}$ is a Sobolev function then $D \mathbf{u}=\nabla \mathbf{u}$ a.e., but the notation $D \mathbf{u}$ will be preferred. The set of approximate differentiability points of $\mathbf{u}$ is usually called $\Omega_{d}$.

Let us recall the classic area formula of Federer [19], which will play a fundamental role in this paper; the formulation is taken from [34, Prop. 2.6].

Proposition 1 Let $\mathbf{u}: \Omega \rightarrow \mathbb{R}^{n}$ be approximately differentiable in almost all $\Omega$ and call $\Omega_{d}$ the set of points of approximate differentiability of $\mathbf{u}$. Then, for any measurable set $A \subset \Omega$ and any measurable functions $\varphi: \mathbb{R}^{n} \rightarrow \mathbb{R}$ and $\psi: A \rightarrow \mathbb{R}$, the function $\tilde{\psi}: \mathbf{u}\left(\Omega_{d} \cap A\right) \rightarrow \mathbb{R}$ given by

$$
\tilde{\psi}(\mathbf{y}):=\sum_{\substack{\mathbf{x} \in \Omega_{d} \cap A \\ \mathbf{u}(\mathbf{x})=\mathbf{y}}} \psi(\mathbf{x})
$$

is measurable and satisfies

$$
\int_{A} \psi(\varphi \circ \mathbf{u})|\operatorname{det} \nabla \mathbf{u}| \mathrm{d} \mathbf{x}=\int_{\mathbf{u}\left(\Omega_{d} \cap A\right)} \tilde{\psi} \varphi \mathrm{d} \mathbf{y}
$$

whenever the integral on the left-hand side exists. 
The Lebesgue $L^{p}$ and Sobolev $W^{1, p}$ spaces are defined in the usual way. So are the set of smooth functions $C^{\infty}$, of bounded variation $B V$ and of special bounded variation $S B V$; see [5], if necessary, for the definitions. The set $C_{c}^{\infty}\left(\Omega, \mathbb{R}^{n}\right)$ denotes the space of $C^{\infty}$ functions with compact support in $\Omega$. We will always indicate the domain and target space, as in, for example, $L^{p}\left(\Omega, \mathbb{R}^{n}\right)$, except if the target space is $\mathbb{R}$, in which case we will simply write $L^{p}(\Omega)$. Weak convergence in Lebesgue or Sobolev spaces is denoted by $\rightarrow$, whereas any other type of convergence (for example, strong or a.e.) is denoted by $\rightarrow$.

Given a measurable set $A \subset \mathbb{R}^{n}$, its characteristic function will be denoted by $\chi_{A}$, and its perimeter by $\operatorname{Per}(A)$, which is defined as

$$
\operatorname{Per}(A):=\sup \left\{\int_{A} \operatorname{div} \mathbf{g}(\mathbf{y}) \mathrm{d} \mathbf{y}: \mathbf{g} \in C_{c}^{\infty}\left(\mathbb{R}^{n}, \mathbb{R}^{n}\right),\|\mathbf{g}\|_{\infty} \leq 1\right\} .
$$

It is well-known that if $A$ is an open set with Lipschitz boundary then $\operatorname{Per}(A)=$ $\mathcal{H}^{n-1}(\partial A)$.

The identity matrix is denoted by $\mathbf{1}$. Given a square matrix $\mathbf{A} \in \mathbb{R}^{n \times n}$, its transpose is denoted by $\mathbf{A}^{T}$, its determinant by $\operatorname{det} \mathbf{A}$, its cofactor matrix by cof $\mathbf{A}$ and is the matrix that satisfies $(\operatorname{det} \mathbf{A}) \mathbf{1}=\mathbf{A}^{T}$ cof $\mathbf{A}$, and its adjoint matrix adj $\mathbf{A}$ is the transpose of cof $\mathbf{A}$. If $\mathbf{A}$ is invertible, its inverse is denoted by $\mathbf{A}^{-1}$, and the transpose of its inverse by $\mathbf{A}^{-T}$.

Let $\mathbf{u}: \Omega \rightarrow \mathbb{R}^{n}$ be approximately differentiable in almost all $\Omega$, and consider the set $\Omega_{d}$ of approximate differentiability points of $\Omega$. For every measurable set $A \subset \Omega$ we define, following [34], the geometric image of $A$ under $\mathbf{u}$ as $\mathbf{u}\left(A \cap \Omega_{d}\right)$. It is denoted by $\operatorname{im}_{\mathrm{G}}(\mathbf{u}, A)$. Although this definition depends of the representative of $\mathbf{u}$, the following property holds: if $\tilde{\mathbf{u}}$ is a function that coincides a.e. with $\mathbf{u}$, and $\tilde{A}$ is a set that coincides a.e. with $A$, then $\operatorname{im}_{\mathrm{G}}(\mathbf{u}, A)$ and $\operatorname{im}_{\mathrm{G}}(\tilde{\mathbf{u}}, \tilde{A})$ also coincide a.e. This can be easily proved by noting that the area formula (Proposition 1) implies the general property

$$
\mathcal{L}^{n}\left(\mathbf{u}\left(N \cap \Omega_{d}\right)\right)=0 \quad \text { whenever } \quad \mathcal{L}^{n}(N)=0 .
$$

Given a measurable set $A \subset \mathbb{R}^{n}$ and a point $\mathbf{x} \in \mathbb{R}^{n}$, we will say that the density of $A$ at $\mathbf{x}$ is 1 when

$$
\lim _{r \searrow 0} \frac{\mathcal{L}^{n}(B(\mathbf{x}, r) \cap A)}{\mathcal{L}^{n}(B(\mathbf{x}, r))}=1 .
$$

We shall make use of the following result of [34, Lemma 2.5].

Lemma 1 Let $\mathbf{u}$ be approximately differentiable in almost all $\Omega$ and suppose that $\operatorname{det} \nabla \mathbf{u}(\mathbf{x}) \neq 0$ a.e. Then there exists a set $\Omega_{0} \subset \Omega$ of full measure in $\Omega$ such that for every $\mathbf{x} \in \Omega_{0}$ and every measurable set $A \subset \Omega$, the density of $\operatorname{im}_{\mathrm{G}}(\mathbf{u}, A)$ at $\mathbf{u}(\mathbf{x})$ is 1 whenever the density of $A$ at $\mathbf{x}$ is 1 .

\section{Surface energy and invertibility of limits of a.e. one-to-one maps}

As was shown in the seminal paper of Ball [6], the weak continuity of the determinant of the deformation gradient is crucial for establishing the existence of minimizers in nonlinear elasticity. However, when the functional space where the problem is formulated allows for cavitation, it is known from the counterexample of Ball and Murat [10] that the above continuity property does not hold. Müller and Spector [34] pointed out that their counterexample, which consists of a sequence of deformations that create 
more and more cavities, could be prevented by including an extra term in the energy that penalizes the creation of new surface. More precisely, they added to the elastic energy a constant multiple of the perimeter of the geometric image of the deformation, which in the case of cavitation should measure the area of the surface of all the cavities created. This, together with other considerations regarding invertibility, allowed them to establish their existence theory of cavitation.

As can be seen in the main convergence result of Müller and Spector [34, Lemma 4.1], in order to prove the weak continuity of the determinants, they require to know that not only all deformations of the sequence have to be one-to-one a.e., but also their limit. One might expect that the weak limit $\mathbf{u}$ of a sequence $\left\{\mathbf{u}_{j}\right\}_{j \in \mathbb{N}}$ of oneto-one a.e. maps satisfying $\operatorname{det} \nabla \mathbf{u}_{j}>0$ and $\operatorname{det} \nabla \mathbf{u}>0$ a.e. is also one-to-one a.e., but they constructed a counterexample showing that this is not true. To overcome this difficulty, they introduced a new invertibility condition, called (INV) and based on the topological degree, upon which their existence theory is built.

In the above-mentioned counterexample, as well as in that of Ball and Murat [10], the total surface energy due to the creation of cavities goes to infinity as $j \rightarrow \infty$. The addition of a surface energy term alone, therefore, should rule out this new counterexample too, without invoking an extra invertibility property. Unfortunately, the surface energy proposed in [34], namely, the perimeter of the geometric image, although it is able to detect the surface of all the cavities created in the example of [10], it fails to do so in the example of [34]. The reason for that is, intuitively, that in the latter example the cavities created are subsequently filled with material from elsewhere in the body, in such a way that their surfaces are no longer part of the reduced boundary of the image of the deformation. (For an exposition of the concept of reduced boundary and other properties of sets of finite perimeter, see, e.g., [19,43,5]).

In the following paragraphs we introduce a new surface energy term that overcomes the difficulties mentioned above (we ought to indicate that [34] already points out a functional that detects the created surface, namely, the mass of the boundary of the current; see Section 7 for a discussion). In order to motivate our definition, let $\mathbf{u}: \Omega \rightarrow \mathbb{R}^{n}$ be an approximately differentiable function in almost all $\Omega$, and let us start by writing down the definition of the perimeter of the set $\operatorname{im}_{\mathrm{G}}(\mathbf{u}, \Omega)$ :

$$
\operatorname{Per}\left(\operatorname{im}_{\mathrm{G}}(\mathbf{u}, \Omega)\right)=\sup \left\{\int_{\operatorname{im}_{\mathrm{G}}(\mathbf{u}, \Omega)} \operatorname{div} \mathbf{g}(\mathbf{y}) \mathrm{d} \mathbf{y}: \mathbf{g} \in C_{c}^{\infty}\left(\mathbb{R}^{n}, \mathbb{R}^{n}\right),\|\mathbf{g}\|_{\infty} \leq 1\right\}
$$

Suppose $\operatorname{det} \nabla \mathbf{u}>0$ a.e. and $\mathbf{u}$ is one-to-one a.e., and consider now the previous expression in the reference configuration

$$
\sup \left\{\int_{\Omega} \operatorname{div} \mathbf{g}(\mathbf{u}(\mathbf{x})) \operatorname{det} \nabla \mathbf{u}(\mathbf{x}) \mathrm{d} \mathbf{x}: \mathbf{g} \in C_{c}^{\infty}\left(\mathbb{R}^{n}, \mathbb{R}^{n}\right),\|\mathbf{g}\|_{\infty} \leq 1\right\}
$$

Suppose for a moment that $\partial \Omega$ is regular enough and that $\mathbf{u}: \Omega \rightarrow \mathbb{R}^{n}$ is a diffeomorphism; then, integrating by parts and using the identity

$$
\operatorname{Div}[\operatorname{adj} \nabla \mathbf{u}(\mathbf{x}) \mathbf{g}(\mathbf{u}(\mathbf{x}))]=\operatorname{div} \mathbf{g}(\mathbf{u}(\mathbf{x})) \operatorname{det} \nabla \mathbf{u}(\mathbf{x})
$$


expression (6) becomes

$$
\begin{aligned}
& \sup \left\{\int_{\Omega} \operatorname{Div}[\operatorname{adj} \nabla \mathbf{u}(\mathbf{x}) \mathbf{g}(\mathbf{u}(\mathbf{x}))] \mathrm{d} \mathbf{x}: \mathbf{g} \in C_{c}^{\infty}\left(\mathbb{R}^{n}, \mathbb{R}^{n}\right),\|\mathbf{g}\|_{\infty} \leq 1\right\} \\
= & \sup \left\{\int_{\partial \Omega} \mathbf{g}(\mathbf{u}(\mathbf{x})) \cdot(\operatorname{cof} \nabla \mathbf{u}(\mathbf{x})) \boldsymbol{\nu}(\mathbf{x}) \mathrm{d} \mathcal{H}^{n-1}(\mathbf{x}): \mathbf{g} \in C_{c}^{\infty}\left(\mathbb{R}^{n}, \mathbb{R}^{n}\right),\|\mathbf{g}\|_{\infty} \leq 1\right\} \\
= & \sup \left\{\int_{\mathbf{u}(\partial \Omega)} \mathbf{g}(\mathbf{y}) \cdot \boldsymbol{\nu}(\mathbf{y}) \mathrm{d} \mathcal{H}^{n-1}(\mathbf{y}): \mathbf{g} \in C_{c}^{\infty}\left(\mathbb{R}^{n}, \mathbb{R}^{n}\right),\|\mathbf{g}\|_{\infty} \leq 1\right\},
\end{aligned}
$$

where $\boldsymbol{\nu}$ denotes the outward normal to $\Omega$ in the first instance, and to $\mathbf{u}(\Omega)$ in the second one. Consequently,

$$
\mathcal{H}^{n-1}(\partial \mathbf{u}(\Omega))=\operatorname{Per}(\mathbf{u}(\Omega))=\mathcal{H}^{n-1}(\mathbf{u}(\partial \Omega)) .
$$

Of course, this is because in this case $\partial \mathbf{u}(\Omega)=\mathbf{u}(\partial \Omega)$. In contrast, when cavities or cracks are created, this does not hold, because, as well as the image of $\partial \Omega$, the boundary of $\operatorname{im}_{\mathrm{G}}(\mathbf{u}, \Omega)$ also contains the surface of the created cavities and the cracks, i.e., the newly created surface.

When singularities are allowed, what fails in the previous argument is that identity (7) does not hold. In fact, the expression of the left-hand side is not even well-defined. Nevertheless, under mild assumptions on $\mathbf{u}$, it is meaningful in the sense of distributions. This gives rise to the following definition.

Definition 1 Let $\mathbf{u}: \Omega \rightarrow \mathbb{R}^{n}$ be approximately differentiable in almost all $\Omega$. Suppose that $\operatorname{det} \nabla \mathbf{u} \in L_{\mathrm{loc}}^{1}(\Omega)$ and $\operatorname{cof} \nabla \mathbf{u} \in L_{\mathrm{loc}}^{1}\left(\Omega, \mathbb{R}^{n \times n}\right)$. For every $\mathbf{g} \in C_{c}^{\infty}\left(\mathbb{R}^{n}, \mathbb{R}^{n}\right)$ and $\phi \in C_{c}^{\infty}(\Omega)$, define

$$
\overline{\mathcal{E}}_{\mathbf{u}}(\phi, \mathbf{g}):=\int_{\Omega}[\mathbf{g}(\mathbf{u}(\mathbf{x})) \cdot(\operatorname{cof} \nabla \mathbf{u}(\mathbf{x})) D \phi(\mathbf{x})+\operatorname{det} \nabla \mathbf{u}(\mathbf{x}) \phi(\mathbf{x})(\operatorname{div} \mathbf{g})(\mathbf{u}(\mathbf{x}))] \mathrm{d} \mathbf{x}
$$

and

$$
\overline{\mathcal{E}}(\mathbf{u}):=\sup \left\{\overline{\mathcal{E}}_{\mathbf{u}}(\phi, \mathbf{g}): \mathbf{g} \in C_{c}^{\infty}\left(\mathbb{R}^{n}, \mathbb{R}^{n}\right), \phi \in C_{c}^{\infty}(\Omega),\|\phi\|_{\infty} \leq 1,\|\mathbf{g}\|_{\infty} \leq 1\right\} .
$$

Following the notation of Definition 1, note that

$$
-\overline{\mathcal{E}}_{\mathbf{u}}(\phi, \mathbf{g})=\langle\operatorname{Div}(\operatorname{adj} \nabla \mathbf{u}) \mathbf{g} \circ \mathbf{u}-(\operatorname{div} \mathbf{g}) \circ \mathbf{u} \operatorname{det} \nabla \mathbf{u}, \phi\rangle
$$

in the sense of distributions. Therefore, the energy $\overline{\mathcal{E}}(\mathbf{u})$, which quantifies the failure of condition (7), also quantifies the failure of equality $\partial \mathbf{u}(\Omega)=\mathbf{u}(\partial \Omega)$, thus detecting the creation of new surface.

Clearly, $\overline{\mathcal{E}}(\mathbf{u})=0$ if $\mathbf{u}$ is smooth. In fact, the following two propositions hold. Proposition 2 is proved in Müller [32, Lemma 2], whereas Proposition 3 is due to Müller, Tang and Yan [35, Th. 3.2].

Proposition 2 Let $\mathbf{u} \in W^{1, p}\left(\Omega, \mathbb{R}^{n}\right)$ satisfy $\operatorname{cof} D \mathbf{u} \in L^{q}\left(\Omega, \mathbb{R}^{n \times n}\right)$ for some $p \geq$ $n-1$ and $q \geq \frac{p}{p-1}$. Then $\overline{\mathcal{E}}(\mathbf{u})=0$.

Proposition 3 Let $\mathbf{u} \in W^{1, p}\left(\Omega, \mathbb{R}^{n}\right)$ satisfy cof $D \mathbf{u} \in L^{q}\left(\Omega, \mathbb{R}^{n \times n}\right)$ for some $p \geq$ $n-1$ and $q \geq \frac{n}{n-1}$. Then $\operatorname{det} D \mathbf{u} \in L^{\frac{q(n-1)}{n}}(\Omega)$ and $\overline{\mathcal{E}}(\mathbf{u})=0$. 
To give an interpretation of $\overline{\mathcal{E}}$ as a surface energy, in the following result we compute $\overline{\mathcal{E}}(\mathbf{u})$ when $\mathbf{u}$ is a deformation that has cracks and creates cavities, but it is smooth elsewhere.

Proposition 4 Let $\Omega$ be a bounded open set of $\mathbb{R}^{n}$. Let $K_{1}, \ldots, K_{M}$ be a finite number of disjoint compact subsets of $\Omega$ of zero measure. Define

$$
\Omega_{1}:=\Omega \backslash \bigcup_{i=1}^{M} K_{i}
$$

Let $\mathbf{u}: \Omega_{1} \rightarrow \mathbb{R}^{n}$ be a $C^{1}$ diffeomorphism such that $\operatorname{det} D \mathbf{u}>0$. Suppose also that

i) $\mathbf{u}$ can be extended continuously to $\partial \Omega \cup \Omega_{1}$, and call $\mathbf{u}$ its extension.

ii) $\mathbf{u}^{-1}: \mathbf{u}\left(\Omega_{1}\right) \rightarrow \mathbb{R}^{n}$ can be extended continuously to $\overline{\mathbf{u}\left(\Omega_{1}\right)}$, and call $\mathbf{u}^{-1}$ its extension.

iii) $\mathbf{u}(\partial \Omega) \cap \mathbf{u}\left(\Omega_{1}\right)=\varnothing$.

iv) $\mathbf{u}\left(\Omega_{1}\right)$ has a Lipschitz boundary.

For each $i=1, \ldots, M$ define $\Gamma_{i}:=\left\{\mathbf{y} \in \partial \mathbf{u}\left(\Omega_{1}\right): \mathbf{u}^{-1}(\mathbf{y}) \in K_{i}\right\}$. Then

$$
\partial \mathbf{u}\left(\Omega_{1}\right)=\mathbf{u}(\partial \Omega) \cup \bigcup_{i=1}^{M} \Gamma_{i}
$$

the union being disjoint. Moreover, denoting by $\boldsymbol{\nu}$ the outward normal to $\mathbf{u}\left(\Omega_{1}\right)$, we have that for all $\phi \in C_{c}^{\infty}(\Omega)$ and $\mathbf{g} \in C_{c}^{\infty}\left(\mathbb{R}^{n}, \mathbb{R}^{n}\right)$,

$$
\overline{\mathcal{E}}_{\mathbf{u}}(\phi, \mathbf{g})=\sum_{i=1}^{M} \int_{\Gamma_{i}} \phi\left(\mathbf{u}^{-1}(\mathbf{y})\right) \mathbf{g}(\mathbf{y}) \cdot \boldsymbol{\nu}(\mathbf{y}) \mathrm{d} \mathcal{H}^{n-1}(\mathbf{y})
$$

and

$$
\overline{\mathcal{E}}(\mathbf{u}) \leq \sum_{i=1}^{M} \mathcal{H}^{n-1}\left(\Gamma_{i}\right)=\operatorname{Per}\left(\operatorname{im}_{\mathrm{G}}(\mathbf{u}, \Omega)\right)-\mathcal{H}^{n-1}(\mathbf{u}(\partial \Omega))
$$

If, in addition, $\mathbf{u}\left(\Omega_{1}\right)$ has a $C^{2}$ boundary then

$$
\overline{\mathcal{E}}(\mathbf{u})=\operatorname{Per}\left(\operatorname{im}_{\mathrm{G}}(\mathbf{u}, \Omega)\right)-\mathcal{H}^{n-1}(\mathbf{u}(\partial \Omega)) .
$$

Proof First we prove that (8) holds. The set $\mathbf{u}(\partial \Omega)$ is contained in $\partial \mathbf{u}\left(\Omega_{1}\right)$ by i), iii) and the fact that $\mathbf{u}\left(\Omega_{1}\right)$ is open. The inclusion $\partial \mathbf{u}\left(\Omega_{1}\right) \subset \mathbf{u}(\partial \Omega) \cup \bigcup_{i=1}^{M} \Gamma_{i}$ can be proved similarly by using also ii) and the equality $\partial \Omega_{1}=\partial \Omega \cup \bigcup_{i=1}^{M} K_{i}$. The union of the right-hand side of $(8)$ is disjoint because so are $K_{1}, \ldots, K_{M}, \partial \Omega$.

Let $\Omega_{d}$ denote the set of approximately differentiable points of $\mathbf{u}$. Since $\mathbf{u}$ is differentiable in $\Omega_{1}$, clearly $\Omega_{1} \subset \Omega_{d}$. Moreover, $\Omega_{d} \backslash \Omega_{1}$ is contained in $\Omega \backslash \Omega_{1}$, which has zero measure. Thus, the sets $\mathbf{u}\left(\Omega_{1}\right)$ and $\operatorname{im}_{\mathrm{G}}(\mathbf{u}, \Omega)$ coincide a.e., and consequently they have the same perimeter. 


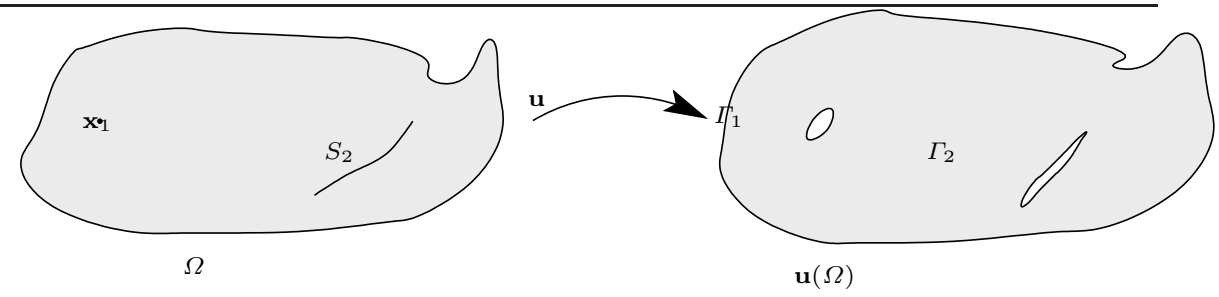

Fig. 1 Deformation $\mathbf{u}$ that is smooth except for a crack and the creation of a cavity.

Now let $\phi \in C_{c}^{\infty}(\Omega)$ and $\mathbf{g} \in C_{c}^{\infty}\left(\mathbb{R}^{n}, \mathbb{R}^{n}\right)$. Changing variables (i.e., using Proposition 1 ) and integrating by parts (which is possible thanks to iv)), we obtain

$$
\begin{aligned}
& \int_{\Omega} \mathbf{g}(\mathbf{u}(\mathbf{x})) \cdot(\operatorname{cof} \nabla \mathbf{u}(\mathbf{x})) D \phi(\mathbf{x}) \mathrm{d} \mathbf{x} \\
= & \int_{\mathbf{u}\left(\Omega_{1}\right)} \mathbf{g}(\mathbf{y}) \cdot\left(D \mathbf{u}\left(\mathbf{u}^{-1}(\mathbf{y})\right)\right)^{-T} D \phi\left(\mathbf{u}^{-1}(\mathbf{y})\right) \mathrm{d} \mathbf{y} \\
= & \int_{\mathbf{u}\left(\Omega_{1}\right)} \mathbf{g}(\mathbf{y}) \cdot D\left(\phi \circ \mathbf{u}^{-1}\right)(\mathbf{y}) \mathrm{d} \mathbf{y} \\
= & -\int_{\mathbf{u}\left(\Omega_{1}\right)} \phi\left(\mathbf{u}^{-1}(\mathbf{y})\right) \operatorname{div} \mathbf{g}(\mathbf{y}) \mathrm{d} \mathbf{y}+\int_{\partial \mathbf{u}\left(\Omega_{1}\right)} \phi\left(\mathbf{u}^{-1}(\mathbf{y})\right) \mathbf{g}(\mathbf{y}) \cdot \boldsymbol{\nu}(\mathbf{y}) \mathrm{d} \mathcal{H}^{n-1}(\mathbf{y}),
\end{aligned}
$$

where $\boldsymbol{\nu}$ is the outward normal to $\mathbf{u}\left(\Omega_{1}\right)$. A further change of variables and (8) show (9). Taking suprema in (9) in functions that satisfy $\|\phi\|_{\infty} \leq 1$ and $\|\mathbf{g}\|_{\infty} \leq 1$, we obtain the inequality of (10). The equality of (10) is due to iv), (8) and the fact that $\operatorname{Per}\left(\mathbf{u}\left(\Omega_{1}\right)\right)=\operatorname{Per}\left(\operatorname{im}_{\mathrm{G}}(\mathbf{u}, \Omega)\right)$.

Finally, if $\mathbf{u}\left(\Omega_{1}\right)$ has a $C^{2}$ boundary, then the normal $\boldsymbol{\nu}$ in (9) is of class $C^{1}$ in $\bigcup_{i=1}^{M} \Gamma_{i}$. Consequently, we can find a $\bar{\phi} \in C_{c}^{\infty}(\Omega)$ and a $\overline{\mathbf{g}} \in C_{c}^{1}\left(\mathbb{R}^{n}, \mathbb{R}^{n}\right)$ such that $\|\bar{\phi}\|_{\infty}=\|\overline{\mathbf{g}}\|_{\infty}=1$, the function $\bar{\phi}$ equals 1 in $\bigcup_{i=1}^{M} K_{i}$, and $\overline{\mathbf{g}}$ equals $\boldsymbol{\nu}$ in $\bigcup_{i=1}^{M} \Gamma_{i}$. Since the supremum in the definition of $\overline{\mathcal{E}}(\mathbf{u})$ (Definition 1) does not change when we take test functions $\mathrm{g}$ in $C_{c}^{1}\left(\mathbb{R}^{n}, \mathbb{R}^{n}\right)$, we conclude that

$$
\overline{\mathcal{E}}(\mathbf{u}) \geq \overline{\mathcal{E}}_{\mathbf{u}}(\bar{\phi}, \overline{\mathbf{g}})=\sum_{i=1}^{M} \mathcal{H}^{n-1}\left(\Gamma_{i}\right),
$$

so showing (11).

An instructive example in Proposition 4 is a deformation $\mathbf{u}$ that creates cavities at $\mathbf{x}_{1}, \ldots, \mathbf{x}_{p}$ and has cracks along the surfaces $S_{p+1}, \ldots, S_{M}$, but it is smooth elsewhere. Then the natural choice of $K_{i}$ is

$$
K_{i}=\left\{\mathbf{x}_{i}\right\}, \quad i=1, \ldots, p ; \quad K_{i}=S_{i}, \quad i=p+1, \ldots, M .
$$

This is illustrated in Figure 1 in the particular case where $p=1$ and $M=2$.

As equation (11) shows, $\overline{\mathcal{E}}(\mathbf{u})$ provides the area of the surface created by $\mathbf{u}$. Consequently, the term $\overline{\mathcal{E}}(\mathbf{u})$, unlike $\operatorname{Per}\left(\operatorname{im}_{\mathrm{G}}(\mathbf{u}, \Omega)\right)$, does not include $\mathcal{H}^{n-1}(\mathbf{u}(\partial \Omega))$, which in fact we believe should not be part of the surface energy, since it does not correspond to the creation of new surface. The main difference, though, between $\overline{\mathcal{E}}(\mathbf{u})$ and $\operatorname{Per}\left(\operatorname{im}_{\mathrm{G}}(\mathbf{u}, \Omega)\right)$ is that the former gives correctly the area of the created surface, even 


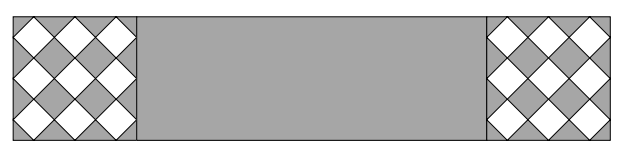

Fig. 2 Deformation $\mathbf{g}_{j}$.

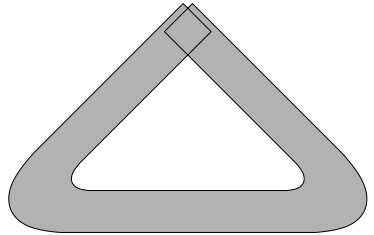

(a)

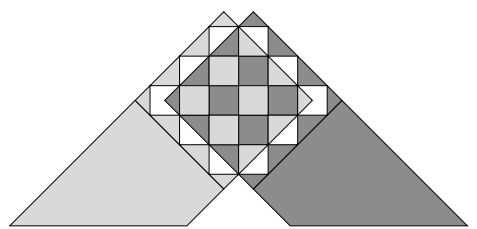

(b)

Fig. 3 Deformations $\mathbf{b}_{j}$ and $\mathbf{u}_{j}$.

in those situations where two pieces of created surface are put in contact. In particular, it measures the (1-dimensional) 'surface' created by the deformations in the example of Müller and Spector [34, Sect. 11], as we shall see in the following paragraphs.

Fix $L \gg 1$ and define $\Omega:=(-L-1, L+1) \times(-1,1)$. In the before-mentioned example a function $\mathbf{u}_{j}: \Omega \rightarrow \mathbb{R}^{2}$ is constructed, for each $j$ odd, such that $\mathbf{u}_{j} \in$ $W^{1, p}\left(\Omega, \mathbb{R}^{2}\right)$ and $\operatorname{det} \nabla \mathbf{u}_{j} \in L^{p}(\Omega)$ for all $p \in[1,2)$. Each $\mathbf{u}_{j}$ is the composition of two maps: $\mathbf{u}_{j}=\mathbf{b}_{j} \circ \mathbf{g}_{j}$. The map $\mathbf{g}_{j}$ is depicted in Figure 2, and deforms the rectangle $\Omega$ into a rectangle with holes. In the subrectangle $(-L+1, L-1) \times(-1,1)$ the deformation $\mathbf{g}_{j}$ is the identity, while in the square $(-L-1,-L+1) \times(-1,1)$ the deformation creates $j^{2}$ square-shaped cavities, as shown in Figure 2. An analogous construction occurs in the square $(L-1, L+1) \times(-1,1)$. This deformation $\mathbf{g}_{j}$ is then composed with a Lipschitz transformation $\mathbf{b}_{j}$ (shown in Figure 3(a)), which bends the rectangle of Figure 2 from the ends. In the deformed configuration of $\mathbf{u}_{j}$ (depicted in Figure 3(b)), the holes created in one end of the deformation have been filled by material from the other end.

We are going to see that $\overline{\mathcal{E}}\left(\mathbf{u}_{j}\right)$ equals the sum of the perimeters of the $2 j^{2}$ squares created by cavitation. This is an essential difference with $\operatorname{Per}\left(\operatorname{im}_{\mathrm{G}}\left(\mathbf{u}_{j}, \Omega\right)\right)$, which equals $\mathcal{H}^{1}\left(\partial \mathbf{u}_{j}(\Omega)\right)$, and hence has two parts: the $\mathcal{H}^{1}$ measure of $\mathbf{u}_{j}(\partial \Omega)$, which is not created surface, and the $\mathcal{H}^{1}$ measure of $\partial \mathbf{u}_{j}(\Omega) \backslash \mathbf{u}_{j}(\partial \Omega)$, which is a small part of the created surface due to the special way that the end squares of Figure $3(\mathrm{~b})$ have been interlaced. Thus, Per $\left(\operatorname{im}_{\mathrm{G}}\left(\mathbf{u}_{j}, \Omega\right)\right)$ does not detect the surface created by cavitation.

Fix $j$ and define the sets $N_{j}, A_{j}^{-}$and $A_{j}^{+}$as

$$
N_{j}:=\left\{-\frac{j-1}{j}, \ldots,-\frac{2}{j}, 0, \frac{2}{j}, \frac{4}{j}, \ldots, \frac{j-1}{j}\right\}, \quad A_{j}^{ \pm}:=\left\{( \pm L+p, q): p, q \in N_{j}\right\} .
$$

The function $\mathbf{u}_{j}$ is locally Lipschitz and one-to-one in $\Omega_{1}:=\Omega \backslash\left(A_{j}^{-} \cup A_{j}^{+}\right)$. Let $\phi \in C_{c}^{\infty}(\Omega)$ and $\mathbf{g} \in C_{c}^{\infty}\left(\mathbb{R}^{2}, \mathbb{R}^{2}\right)$. As in $(12)$,

$$
\int_{\Omega} \mathbf{g}\left(\mathbf{u}_{j}(\mathbf{x})\right) \cdot\left(\operatorname{cof} \nabla \mathbf{u}_{j}(\mathbf{x})\right) D \phi(\mathbf{x}) \mathrm{d} \mathbf{x}=\int_{\mathbf{u}_{j}\left(\Omega_{1}\right)} \mathbf{g}(\mathbf{y}) \cdot D\left(\phi \circ \mathbf{u}_{j}^{-1}\right)(\mathbf{y}) \mathrm{d} \mathbf{y}
$$


Now define $\Omega_{1}^{-}:=\Omega_{1} \cap[(-\infty, 0) \times \mathbb{R}]$ and $\Omega_{1}^{+}:=\Omega_{1} \cap[(0, \infty) \times \mathbb{R}]$, let $\mathbf{u}_{j}^{ \pm}$be the restriction of $\mathbf{u}_{j}$ to $\Omega_{1}^{ \pm}$, and note that $\left(\mathbf{u}_{j}^{ \pm}\right)^{-1}: \mathbf{u}_{j}\left(\Omega_{1}^{ \pm}\right) \rightarrow \mathbb{R}^{2}$ can be extended continuously to $\overline{\mathbf{u}_{j}\left(\Omega_{1}^{ \pm}\right)}$. Reasoning again as in (12), we have that

$$
\begin{aligned}
\overline{\mathcal{E}}_{\mathbf{u}_{j}}(\phi, \mathbf{g})= & \int_{\partial \mathbf{u}_{j}\left(\Omega_{1}^{-}\right)} \phi\left(\left(\mathbf{u}_{j}^{-}\right)^{-1}(\mathbf{y})\right) \mathbf{g}(\mathbf{y}) \cdot \boldsymbol{\nu}^{-}(\mathbf{y}) \mathrm{d} \mathcal{H}^{1}(\mathbf{y}) \\
& +\int_{\partial \mathbf{u}_{j}\left(\Omega_{1}^{+}\right)} \phi\left(\left(\mathbf{u}_{j}^{+}\right)^{-1}(\mathbf{y})\right) \mathbf{g}(\mathbf{y}) \cdot \nu^{+}(\mathbf{y}) \mathrm{d} \mathcal{H}^{1}(\mathbf{y}),
\end{aligned}
$$

where $\boldsymbol{\nu}^{ \pm}$is the outward normal to $\mathbf{u}\left(\Omega_{1}^{ \pm}\right)$.

Define $\Gamma:=\partial \mathbf{u}\left(\Omega_{1}^{-}\right) \cap \partial \mathbf{u}\left(\Omega_{1}^{+}\right)$and note that $\left(\mathbf{u}_{j}^{-}\right)^{-1}$ and $\left(\mathbf{u}_{j}^{+}\right)^{-1}$ coincide in $\Gamma$, whereas $\nu^{-}=-\nu^{+}$in $\Gamma$; consequently,

$$
\begin{aligned}
\overline{\mathcal{E}}_{\mathbf{u}_{j}}(\phi, \mathbf{g})= & \int_{\partial \mathbf{u}_{j}\left(\Omega_{1}^{-}\right) \backslash \Gamma} \phi\left(\left(\mathbf{u}_{j}^{-}\right)^{-1}(\mathbf{y})\right) \mathbf{g}(\mathbf{y}) \cdot \boldsymbol{\nu}^{-}(\mathbf{y}) \mathrm{d} \mathcal{H}^{1}(\mathbf{y}) \\
& +\int_{\partial \mathbf{u}_{j}\left(\Omega_{1}^{+}\right) \backslash \Gamma} \phi\left(\left(\mathbf{u}_{j}^{+}\right)^{-1}(\mathbf{y})\right) \mathbf{g}(\mathbf{y}) \cdot \nu^{+}(\mathbf{y}) \mathrm{d} \mathcal{H}^{1}(\mathbf{y}) .
\end{aligned}
$$

For each $\mathbf{z} \in A_{j}^{ \pm}$, define $C_{j, \mathbf{z}}^{ \pm}:=\left\{\mathbf{y} \in \partial \mathbf{u}_{j}\left(\Omega_{1}^{ \pm}\right):\left(\mathbf{u}_{j}^{ \pm}\right)^{-1}(\mathbf{y})=\mathbf{z}\right\}$, and observe that $C_{j, \mathbf{z}}^{ \pm}$intuitively corresponds to the surface of the cavity created at the point $\mathbf{z}$. As $\phi$ vanishes on $\partial \Omega$, its is easy to see that

$$
\begin{aligned}
& \overline{\mathcal{E}}_{\mathbf{u}_{j}}(\phi, \mathbf{g}) \\
= & \sum_{\mathbf{z} \in A_{j}^{-}} \phi(\mathbf{z}) \int_{C_{j, \mathbf{z}}^{-}} \mathbf{g}(\mathbf{y}) \cdot \boldsymbol{\nu}^{-}(\mathbf{y}) \mathrm{d} \mathcal{H}^{1}(\mathbf{y})+\sum_{\mathbf{z} \in A_{j}^{+}} \phi(\mathbf{z}) \int_{C_{j, \mathbf{z}}^{+}} \mathbf{g}(\mathbf{y}) \cdot \nu^{+}(\mathbf{y}) \mathrm{d} \mathcal{H}^{1}(\mathbf{y}) .
\end{aligned}
$$

Now we note that

$$
\nu^{-}(\mathbf{y})=-\nu^{+}(\mathbf{y}), \quad \mathbf{y} \in\left(\bigcup_{\mathbf{z} \in A_{j}^{-}} C_{j, \mathbf{z}}^{-}\right) \cap\left(\bigcup_{\mathbf{z} \in A_{j}^{+}} C_{j, \mathbf{z}}^{+}\right) .
$$

From expressions (13) and (14), we can easily see that the supremum in the definition of $\overline{\mathcal{E}}$ (Definition 1 ) is not attained, but its value can be approximated in the following way. Remove from the square $C_{j, \mathbf{z}}^{ \pm}$a small neighbourhood of its vertices, and call this set $D_{j, \mathbf{z}}^{ \pm}$. Take functions $\bar{\phi} \in C_{c}^{\infty}(\Omega)$ and $\overline{\mathbf{g}} \in C_{c}^{\infty}\left(\mathbb{R}^{2}, \mathbb{R}^{2}\right)$ such that $\|\bar{\phi}\|_{\infty}=\|\overline{\mathbf{g}}\|_{\infty}=1$ and

$$
\bar{\phi}(\mathbf{z})= \pm 1, \quad \text { for } \quad \mathbf{z} \in A_{j}^{ \pm} ; \quad \overline{\mathbf{g}}(\mathbf{y})= \pm \boldsymbol{\nu}(\mathbf{y}), \quad \text { for } \mathbf{y} \in D_{j, \mathbf{z}}^{ \pm} \text {and } \mathbf{z} \in A_{j}^{ \pm} .
$$

Thus, we conclude that

$$
\overline{\mathcal{E}}\left(\mathbf{u}_{j}\right)=\sum_{\mathbf{z} \in A_{j}^{-}} \mathcal{H}^{1}\left(C_{j, \mathbf{z}}^{-}\right)+\sum_{\mathbf{z} \in A_{j}^{+}} \mathcal{H}^{1}\left(C_{j, \mathbf{z}}^{+}\right)=8 \sqrt{2} j,
$$

as desired.

As Müller and Spector [34] pointed out, the sequence $\mathbf{u}_{j}$ converges weakly in $W^{1, p}$ for all $p \in[1,2)$ to a function that is not one-to-one a.e., despite every $\mathbf{u}_{j}$ being one-to-one a.e. In order to prevent that undesirable behaviour, they introduced the topological condition (INV). In the following theorem, whose proof uses several ideas of [34], we show that, without the need of any topological condition, this behaviour is also prevented if the energy $\overline{\mathcal{E}}$ of the sequence is bounded. 
Theorem 2 For each $j \in \mathbb{N}$, let $\mathbf{u}_{j}, \mathbf{u}: \Omega \rightarrow \mathbb{R}^{n}$ be approximately differentiable in almost all $\Omega$,

$$
\operatorname{cof} \nabla \mathbf{u}_{j} \in L^{1}\left(\Omega, \mathbb{R}^{n \times n}\right), \quad \operatorname{det} \nabla \mathbf{u}_{j} \in L^{1}(\Omega)
$$

and

$$
\sup _{j \in \mathbb{N}}\left\|\operatorname{cof} \nabla \mathbf{u}_{j}\right\|_{L^{1}\left(\Omega, \mathbb{R}^{n \times n}\right)}<\infty, \quad \sup _{j \in \mathbb{N}} \overline{\mathcal{E}}\left(\mathbf{u}_{j}\right)<\infty .
$$

Suppose that there exists $\theta \in L^{1}(\Omega)$ such that $\theta>0$ a.e., and

$$
\mathbf{u}_{j} \rightarrow \mathbf{u} \text { a.e. } \quad \text { and } \operatorname{det} \nabla \mathbf{u}_{j} \rightarrow \theta \text { in } L^{1}(\Omega)
$$

as $j \rightarrow \infty$. Assume that for each $j \in \mathbb{N}$, the function $\mathbf{u}_{j}$ is one-to-one a.e. with $\operatorname{det} \nabla \mathbf{u}_{j}>0$ a.e. Then

i) $\theta=|\operatorname{det} \nabla \mathbf{u}|$ a.e.

ii) $\mathbf{u}$ is one-to-one a.e.

iii) For every $\mathbf{x} \in \Omega$ there exists $R_{\mathbf{x}} \subset(0, \operatorname{dist}(\mathbf{x}, \partial \Omega))$ such that

$$
\mathcal{L}^{1}\left((0, \operatorname{dist}(\mathbf{x}, \partial \Omega)) \backslash R_{\mathbf{x}}\right)=0
$$

and for each $r \in R_{\mathbf{x}}$,

$$
\operatorname{Per}\left(\operatorname{im}_{\mathrm{G}}(\mathbf{u}, B(\mathbf{x}, r))\right) \leq \liminf _{j \rightarrow \infty} \operatorname{Per}\left(\operatorname{im}_{\mathrm{G}}\left(\mathbf{u}_{j}, B(\mathbf{x}, r)\right)\right)<\infty
$$

and there exists a subsequence $\left\{j_{k}\right\}_{k \in \mathbb{N}}$ such that

$$
\chi_{\mathrm{im}_{\mathrm{G}}\left(\mathbf{u}_{j_{k}}, B(\mathbf{x}, r)\right)} \rightarrow \chi_{\mathrm{im}_{\mathrm{G}}(\mathbf{u}, B(\mathbf{x}, r))} \quad \text { in } L_{\mathrm{loc}}^{1}\left(\mathbb{R}^{n}\right)
$$

as $k \rightarrow \infty$.

Proof Without loss of generality, $\mathbf{u}_{j}$ and $\mathbf{u}$ are the precise representatives of themselves; that is, if for an $\mathbf{x}_{0} \in \Omega$ there exists $\mathbf{a} \in \mathbb{R}^{n}$ such that

$$
\lim _{r \searrow 0} f_{B\left(\mathbf{x}_{0}, r\right)}|\mathbf{u}(\mathbf{x})-\mathbf{a}| \mathrm{d} \mathbf{x}=0
$$

then $\mathbf{a}=\mathbf{u}\left(\mathbf{x}_{0}\right)$, and analogously for $\mathbf{u}_{j}$.

Fix $\mathbf{x}_{0} \in \Omega$ and let $s_{0}:=\operatorname{dist}\left(\mathbf{x}_{0}, \partial \Omega\right)$. For each $j \in \mathbb{N}$, define the function $f_{j}$ : $\left(0, s_{0}\right) \rightarrow \mathbb{R}$ as

$$
f_{j}(r):=\int_{\partial B\left(\mathbf{x}_{0}, r\right)}\left|\operatorname{cof} \nabla \mathbf{u}_{j}\right| \mathrm{d} \mathcal{H}^{n-1}, \quad r \in\left(0, s_{0}\right) .
$$

By Fubini's theorem, for a.e. $r_{0} \in\left(0, s_{0}\right)$ and every $j \in \mathbb{N}$,

a) $\mathbf{u}_{j}$ is approximately differentiable at $\mathcal{H}^{n-1}$-a.e. point of $\partial B\left(\mathbf{x}_{0}, r_{0}\right)$,

b) $f_{j}\left(r_{0}\right)$ is well defined and finite,

c) $\lim _{\varepsilon \rightarrow 0} f_{r_{0}-\varepsilon}^{r_{0}-\frac{\varepsilon}{2}}\left|f_{j}(r)-f_{j}\left(r_{0}\right)\right| \mathrm{d} r=0$. 
Property c) follows, of course, from a version of Lebesgue's differentiation theorem, a proof of which can be found, for example, in [39, Th. 8.8]. By Fatou's lemma and Fubini's theorem,

$$
\int_{0}^{s_{0}} \liminf _{j \rightarrow \infty} f_{j}(r) \mathrm{d} r \leq \liminf _{j \rightarrow \infty}\left\|\operatorname{cof} \nabla \mathbf{u}_{j}\right\|_{L^{1}\left(\Omega, \mathbb{R}^{n \times n}\right)}<\infty .
$$

Therefore, there exists $R_{0} \subset\left(0, s_{0}\right)$ such that $\mathcal{L}^{1}\left(\left(0, s_{0}\right) \backslash R_{0}\right)=0$, and for each $r \in R_{0}$, properties a)-c) above hold for all $j \in \mathbb{N}$, and, in addition,

$$
\liminf _{j \rightarrow \infty} f_{j}(r)<\infty
$$

Fix any $r_{0} \in R_{0}$. For each $\varepsilon \in\left(0, r_{0}\right)$, choose any $C^{\infty}$ function $\varphi_{\varepsilon}:[0, \infty) \rightarrow[0,1]$ such that

$$
\begin{aligned}
& \varphi_{\varepsilon}(t)=1 \text { for each } t \in\left[0, r_{0}-\varepsilon\right) \text { and each } \varepsilon \in\left(0, r_{0}\right), \\
& \varphi_{\varepsilon}(t)=0 \text { for each } t \in\left[r_{0}-\frac{\varepsilon}{2}, \infty\right) \text { and each } \varepsilon \in\left(0, r_{0}\right), \\
& \varepsilon\left\|\varphi_{\varepsilon}^{\prime}\right\|_{\infty} \leq 4 \text { for each } \varepsilon \in\left(0, r_{0}\right) .
\end{aligned}
$$

Call, for simplicity, $B:=B\left(\mathbf{x}_{0}, r_{0}\right)$. For each $\varepsilon \in\left(0, r_{0}\right)$, consider the function $\phi_{\varepsilon} \in$ $C_{c}^{\infty}(\Omega)$ defined as $\phi_{\varepsilon}(\mathbf{x})=\varphi_{\varepsilon}\left(\left|\mathbf{x}-\mathbf{x}_{0}\right|\right)$ for $\mathbf{x} \in B$, and $\phi_{\varepsilon}=0$ in $\Omega \backslash \bar{B}$. Clearly, $\phi_{\varepsilon} \rightarrow \chi_{B}$ pointwise as $\varepsilon \rightarrow 0$.

Take now a $\mathbf{g} \in C_{c}^{\infty}\left(\mathbb{R}^{n}, \mathbb{R}^{n}\right)$ with $\|\mathbf{g}\|_{\infty} \leq 1$. Then, for each $j \in \mathbb{N}$, by Lebesgue's dominated convergence theorem and Proposition 1,

$$
\lim _{\varepsilon \rightarrow 0} \int_{\Omega} \phi_{\varepsilon}(\mathbf{x}) \operatorname{div} \mathbf{g}\left(\mathbf{u}_{j}(\mathbf{x})\right) \operatorname{det} \nabla \mathbf{u}_{j}(\mathbf{x}) \mathrm{d} \mathbf{x}=\int_{\operatorname{im}_{\mathrm{G}}\left(\mathbf{u}_{j}, B\right)} \operatorname{div} \mathbf{g}(\mathbf{y}) \mathrm{d} \mathbf{y} .
$$

According to Definition 1 , for each $\varepsilon \in\left(0, r_{0}\right)$ and $j \in \mathbb{N}$,

$$
\begin{aligned}
& \int_{\Omega} \phi_{\varepsilon}(\mathbf{x}) \operatorname{div} \mathbf{g}\left(\mathbf{u}_{j}(\mathbf{x})\right) \operatorname{det} \nabla \mathbf{u}_{j}(\mathbf{x}) \mathrm{d} \mathbf{x} \\
= & \overline{\mathcal{E}}_{\mathbf{u}_{j}}\left(\phi_{\varepsilon}, \mathbf{g}\right)-\int_{\Omega} \mathbf{g}\left(\mathbf{u}_{j}(\mathbf{x})\right) \cdot \operatorname{cof} \nabla \mathbf{u}_{j}(\mathbf{x}) D \phi_{\varepsilon}(\mathbf{x}) \mathrm{d} \mathbf{x},
\end{aligned}
$$

and hence, taking limits when $\varepsilon \rightarrow 0$,

$$
\int_{\operatorname{im}_{\mathrm{G}}\left(\mathbf{u}_{j}, B\right)} \operatorname{div} \mathbf{g}(\mathbf{y}) \mathrm{d} \mathbf{y} \leq \overline{\mathcal{E}}\left(\mathbf{u}_{j}\right)+\liminf _{\varepsilon \rightarrow 0}\left|\int_{\Omega} \mathbf{g}\left(\mathbf{u}_{j}(\mathbf{x})\right) \cdot \operatorname{cof} \nabla \mathbf{u}_{j}(\mathbf{x}) D \phi_{\varepsilon}(\mathbf{x}) \mathrm{d} \mathbf{x}\right| .
$$

Now for each $\varepsilon \in\left(0, r_{0}\right)$ and $j \in \mathbb{N}$, by Fubini's theorem,

$$
\begin{aligned}
& \left|\int_{\Omega} \mathbf{g}\left(\mathbf{u}_{j}(\mathbf{x})\right) \cdot\left(\operatorname{cof} \nabla \mathbf{u}_{j}(\mathbf{x})\right) D \phi_{\varepsilon}(\mathbf{x}) \mathrm{d} \mathbf{x}\right| \\
\leq & \int_{r_{0}-\varepsilon}^{r_{0}-\frac{\varepsilon}{2}} \frac{\varepsilon}{2}\left|\varphi_{\varepsilon}^{\prime}(r)\right|\left|\int_{\partial B\left(\mathbf{x}_{0}, r\right)} \mathbf{g}\left(\mathbf{u}_{j}(\mathbf{x})\right) \cdot \operatorname{cof} \nabla \mathbf{u}_{j}(\mathbf{x}) \frac{\mathbf{x}-\mathbf{x}_{0}}{\left|\mathbf{x}-\mathbf{x}_{0}\right|} \mathrm{d} \mathcal{H}^{n-1}(\mathbf{x})\right| \mathrm{d} r \\
\leq & 2 f_{r_{0}-\varepsilon}^{r_{0}-\frac{\varepsilon}{2}} f_{j}(r) \mathrm{d} r
\end{aligned}
$$

and hence

$$
\limsup _{\varepsilon \rightarrow 0}\left|\int_{\Omega} \mathbf{g}\left(\mathbf{u}_{j}(\mathbf{x})\right) \cdot\left(\operatorname{cof} \nabla \mathbf{u}_{j}(\mathbf{x})\right) D \phi_{\varepsilon}(\mathbf{x}) \mathrm{d} \mathbf{x}\right| \leq 2 f_{j}\left(r_{0}\right)
$$


Thus,

$$
\int_{\operatorname{im}_{\mathrm{G}}\left(\mathbf{u}_{j}, B\right)} \operatorname{div} \mathbf{g}(\mathbf{y}) \mathrm{d} \mathbf{y} \leq \overline{\mathcal{E}}\left(\mathbf{u}_{j}\right)+2 f_{j}\left(r_{0}\right)
$$

and, hence, according to the definition of perimeter,

$$
\operatorname{Per}\left(\operatorname{im}_{\mathrm{G}}\left(\mathbf{u}_{j}, B\right)\right) \leq \overline{\mathcal{E}}\left(\mathbf{u}_{j}\right)+2 f_{j}\left(r_{0}\right) .
$$

Taking limits when $j \rightarrow \infty$, thanks to (15), we obtain that

$$
\liminf _{j \rightarrow \infty} \operatorname{Per}\left(\operatorname{im}_{\mathrm{G}}\left(\mathbf{u}_{j}, B\right)\right)<\infty .
$$

Thus, by the compact embedding of $B V_{\text {loc }}$ into $L_{\text {loc }}^{1}$, for a subsequence (not relabelled), there exists a measurable set $V \subset \mathbb{R}^{n}$ such that $\chi_{\mathrm{im}_{\mathrm{G}}\left(\mathbf{u}_{j}, B\right)} \rightarrow \chi_{V}$ in $L_{\mathrm{loc}}^{1}\left(\mathbb{R}^{n}\right)$ as $j \rightarrow \infty$, and $\operatorname{Per}(V) \leq \liminf _{j \rightarrow \infty} \operatorname{Per}\left(\operatorname{im}_{\mathrm{G}}\left(\mathbf{u}_{j}, B\right)\right)$.

Let $\varphi \in C\left(\mathbb{R}^{n}\right)$ have compact support. By Proposition 1 , for all $j \in \mathbb{N}$,

$$
\int_{\operatorname{im}_{\mathrm{G}}\left(\mathbf{u}_{j}, B\right)} \varphi(\mathbf{y}) \mathrm{d} \mathbf{y}=\int_{B} \varphi\left(\mathbf{u}_{j}(\mathbf{x})\right) \operatorname{det} \nabla \mathbf{u}_{j}(\mathbf{x}) \mathrm{d} \mathbf{x} .
$$

Using a standard convergence result (see, e.g., [40, Lemma 6.7]), we obtain

$$
\int_{\mathbb{R}^{n}} \varphi(\mathbf{y}) \chi_{V}(\mathbf{y}) \mathrm{d} \mathbf{y}=\int_{B} \varphi(\mathbf{u}(\mathbf{x})) \theta(\mathbf{x}) \mathrm{d} \mathbf{x} .
$$

The latter equality, which is true for any $\varphi \in C\left(\mathbb{R}^{n}\right)$ of compact support, is also valid for any Borel function $\varphi: \mathbb{R}^{n} \rightarrow \mathbb{R}$.

Now we show that $\operatorname{det} \nabla \mathbf{u}(\mathbf{x}) \neq 0$ for a.e. $\mathbf{x} \in \Omega$. Let $\Omega_{d}$ be the set of approximate differentiablity points of $\mathbf{u}$, which by assumption has full measure in $\Omega$. Let $Z$ be the set of $\mathbf{x} \in \Omega_{d}$ such that $\operatorname{det} \nabla \mathbf{u}(\mathbf{x})=0$. Property (5) shows that $\mathcal{L}^{n}(\mathbf{u}(Z))=0$. Thus, there exists a Borel set $U$ containing $\mathbf{u}(Z)$ such that $\mathcal{L}^{n}(U)=0$. Applying (16) with $\varphi=\chi_{U}$, we obtain that $\int_{B \cap Z} \theta \mathrm{d} \mathbf{x}=0$ and $\mathcal{L}^{n}(B \cap Z)=0$. Define $\mathcal{B}:=\{B(\mathbf{x}, r): \mathbf{x} \in$ $\left.\Omega, r \in R_{\mathbf{x}}\right\}$. We have thus shown that $\mathcal{L}^{n}(B \cap Z)=0$ for all $B \in \mathcal{B}$, hence $\mathcal{L}^{n}(Z)=0$.

Consider the set $\Omega_{0}$ of Lemma 1 , and define $\Omega_{1}$ as the set of $\mathbf{x} \in \Omega_{d} \cap \Omega_{0}$ such that $\operatorname{det} \nabla \mathbf{u}(\mathbf{x}) \neq 0$ and $\theta(\mathbf{x})>0$. We have shown that $\Omega_{1}$ has full measure in $\Omega$. Again by Proposition 1 , the function $\tilde{\psi}: \mathbb{R}^{n} \rightarrow \mathbb{R}$ defined by

$$
\tilde{\psi}(\mathbf{y}):=\sum_{\substack{\mathbf{x} \in B \cap \Omega_{1} \\ \mathbf{u}(\mathbf{x})=\mathbf{y}}} \frac{\theta(\mathbf{x})}{|\operatorname{det} \nabla \mathbf{u}(\mathbf{x})|}, \quad \mathbf{y} \in \mathbb{R}^{n}
$$

satisfies that for any measurable function $\varphi: \mathbb{R}^{n} \rightarrow \mathbb{R}$,

$$
\int_{B} \varphi(\mathbf{u}(\mathbf{x})) \theta(\mathbf{x}) \mathrm{d} \mathbf{x}=\int_{\mathbb{R}^{n}} \varphi(\mathbf{y}) \tilde{\psi}(\mathbf{y}) \chi_{\mathrm{im}_{\mathrm{G}}(\mathbf{u}, B)}(\mathbf{y}) \mathrm{d} \mathbf{y}
$$

Equalities (16) and (17) show that $\chi_{V}=\tilde{\psi} \chi_{\operatorname{im}_{\mathrm{G}}(\mathbf{u}, B)}$ a.e. Since $\theta>0$ a.e., necessarily $\chi_{V}=\chi_{\mathrm{im}_{\mathrm{G}}(\mathbf{u}, B)}$ a.e. and

$$
\tilde{\psi}(\mathbf{y})=1 \quad \text { a.e. } \quad \mathbf{y} \in \operatorname{im}_{\mathrm{G}}(\mathbf{u}, B)
$$

This finishes the proof of part iii) of the theorem. 
Now let $B_{1}$ and $B_{2}$ be two disjoint balls in $\mathcal{B}$. By iii), there exists a subsequence (not relabelled) such that $\chi_{\mathrm{im}_{\mathrm{G}}\left(\mathbf{u}_{j}, B_{i}\right)} \rightarrow \chi_{\mathrm{im}_{\mathrm{G}}\left(\mathbf{u}, B_{i}\right)}$ a.e. as $j \rightarrow \infty$, for $i=1,2$. Since, for each $j \in \mathbb{N}$, the function $\mathbf{u}_{j}$ is one-to-one a.e., we have that

$$
\chi_{\mathrm{im}_{\mathrm{G}}\left(\mathbf{u}_{j}, B_{1}\right)}(\mathbf{y})+\chi_{\mathrm{im}_{\mathrm{G}}\left(\mathbf{u}_{j}, B_{2}\right)}(\mathbf{y}) \leq 1
$$

for a.e. $\mathbf{y} \in \mathbb{R}^{n}$; indeed, let $V_{j}$ be the set of differentiability points of $\mathbf{u}_{j}$, and $U_{j}$ a set of full measure in $\Omega$ in which $\mathbf{u}_{j}$ is one-to-one. We claim that (19) is true for all $\mathbf{y} \in \mathbb{R}^{n} \backslash \mathbf{u}_{j}\left(V_{j} \backslash U_{j}\right)$, and hence for a.e. $\mathbf{y} \in \mathbb{R}^{n}$, since by (5) the set $\mathbf{u}_{j}\left(V_{j} \backslash U_{j}\right)$ has measure zero. Let $\mathbf{y} \in \operatorname{im}_{\mathrm{G}}\left(\mathbf{u}_{j}, B_{1}\right) \cap \mathrm{im}_{\mathrm{G}}\left(\mathbf{u}_{j}, B_{2}\right)$, so $\mathbf{y}=\mathbf{u}_{j}\left(\mathbf{x}_{1}\right)=\mathbf{u}_{j}\left(\mathbf{x}_{2}\right)$ for some $\mathbf{x}_{i} \in V_{j} \cap B_{i}(i=1,2)$. Since $\mathbf{u}_{j}$ is one-to-one in $U_{j}$, at least one of $\mathbf{x}_{1}, \mathbf{x}_{2}$ does not belong to $U_{j}$, and hence $\mathbf{y} \in \mathbf{u}_{j}\left(V_{j} \backslash U_{j}\right)$. Passing to the limit in (19), we obtain

$$
\chi_{\mathrm{im}_{\mathrm{G}}\left(\mathbf{u}, B_{1}\right)}(\mathbf{y})+\chi_{\mathrm{im}_{\mathrm{G}}\left(\mathbf{u}, B_{2}\right)}(\mathbf{y}) \leq 1
$$

for a.e. $\mathbf{y} \in \mathbb{R}^{n}$, and hence

$$
\mathcal{L}^{n}\left(\operatorname{im}_{\mathrm{G}}\left(\mathbf{u}, B_{1}\right) \cap \operatorname{im}_{\mathrm{G}}\left(\mathbf{u}, B_{2}\right)\right)=0 .
$$

Now we show that $\mathbf{u}$ is one-to-one in $\Omega_{1}$. Let $\mathbf{x}_{1}, \mathbf{x}_{2} \in \Omega_{1}$ be two different points, and assume, for a contradiction, that $\mathbf{u}\left(\mathbf{x}_{1}\right)=\mathbf{u}\left(\mathbf{x}_{2}\right)$. For each $i=1,2$ take $B_{i} \in \mathcal{B}$ such that $\mathbf{x}_{i} \in B_{i}$ and $B_{1} \cap B_{2}=\varnothing$. Then the set $B_{i} \cap \Omega_{1}$ has density 1 at $\mathbf{x}_{i}$, so by Lemma 1 , the set $\mathbf{u}\left(B_{i} \cap \Omega_{1}\right)$ has density 1 at $\mathbf{u}\left(\mathbf{x}_{1}\right)=\mathbf{u}\left(\mathbf{x}_{2}\right)$, a contradiction with (20).

Let $B \in \mathcal{B}$. Since $\mathbf{u}$ is one-to-one in $\Omega_{1}$, from the definition of $\tilde{\psi}$ and (18), we conclude that $\theta(\mathbf{x})=|\operatorname{det} \nabla \mathbf{u}(\mathbf{x})|$ for a.e. $\mathbf{x} \in B$. Since this is true for any $B \in \mathcal{B}$, then $\theta=|\operatorname{det} \nabla \mathbf{u}|$ a.e.

We remark that, in Theorem 2iii), the subsequence $\left\{j_{k}\right\}_{k \in \mathbb{N}}$ depends on $\mathbf{x}$ and $r$.

Theorem 2 improves the results by Ciarlet and Nečas [13] and Tang [41] on the stability of the invertibility condition under the weak limit. It is related, in addition, to the results of Giacomini and Ponsiglione [26, Th. 4.4].

\section{Weak continuity of the determinant}

In Section 3 we derived the energy functional $\overline{\mathcal{E}}$ as a refinement of the surface energy term $\operatorname{Per} \mathbf{u}(\Omega)$ that is, in addition, capable of detecting all the created surface in the counterexample of Müller and Spector [34]. We obtained that functional from the identity

$$
(\operatorname{div} \mathbf{g})(\mathbf{u}(\mathbf{x})) \operatorname{det} D \mathbf{u}(\mathbf{x})=\operatorname{Div}((\operatorname{adj} D \mathbf{u}(\mathbf{x})) \mathbf{g}(\mathbf{u}(\mathbf{x})))
$$

by interpreting the divergence on the right-hand side in a distributional sense. Of course, $(21)$ is itself a generalization of the well-known formula $\operatorname{det} D \mathbf{u}=\frac{1}{n} \operatorname{Div}((\operatorname{adj} D \mathbf{u}) \mathbf{u})$.

We begin this section by pointing out that our energy functional, apart from having the above interpretation, is also related to a well-known object in the theory of currents (see, e.g., $[19,28,29])$. Let $\phi \in C_{c}^{\infty}(\Omega)$ and $\mathbf{g} \in C_{c}^{\infty}\left(\mathbb{R}^{n}, \mathbb{R}^{n}\right)$, and consider the $(n-1)$ differential form $\omega$ defined by

$$
\omega(\mathbf{x}, \mathbf{y}):=\sum_{j=1}^{n}(-1)^{j-1} \phi(\mathbf{x}) g^{j}(\mathbf{y}) \widehat{\mathrm{d} y^{j}},
$$


where the functions $g^{j}$ stand for the coordinates of $\mathbf{g}$. Then, denoting by $G_{\mathbf{u}}$ the current carried by the graph of $\mathbf{u}$, we have that $\overline{\mathcal{E}}_{\mathbf{u}}(\phi, \mathbf{g})=\left\langle\partial G_{\mathbf{u}}, \omega\right\rangle$. For an exposition of differential forms and the theory of currents, as well as for the notation and terminology used, we refer the reader to $[28,29]$.

One of the main features of the theory of currents (in fact, one reason for its success) is that it offers a unified treatment for the reference and the deformed configuration associated to a deformation. This is achieved by using test functions depending in both the $\mathbf{x}$ and the $\mathbf{y}$ variables. Given any $\mathbf{f} \in C_{c}^{\infty}\left(\Omega \times \mathbb{R}^{n}, \mathbb{R}^{n}\right)$, define the differential form

$$
\omega_{\mathbf{f}}(\mathbf{x}, \mathbf{y}):=\sum_{j=1}^{n}(-1)^{j-1} f^{j}(\mathbf{x}, \mathbf{y}) \widehat{\mathrm{d} y^{j}}
$$

where the functions $f^{j}$ denote the coordinates of $\mathbf{f}$. It is clear that the differential form $\omega$ defined in (22) is a particular case of $\omega_{\mathbf{f}}$ corresponding to the choice $\mathbf{f}(\mathbf{x}, \mathbf{y})=\phi(\mathbf{x}) \mathbf{g}(\mathbf{y})$. From the point of view of the theory of currents, therefore, our functional $\overline{\mathcal{E}}_{\mathbf{u}}(\phi, \mathbf{g})$ is just a particular case of a more general object acting really on functions of $2 n$ variables, namely, the linear functional $\mathbf{f} \mapsto\left\langle\partial G_{\mathbf{u}}, \omega_{\mathbf{f}}\right\rangle$. This motivates the following definition.

Definition 2 Let $\mathbf{u}: \Omega \rightarrow \mathbb{R}^{n}$ be approximately differentiable in almost all $\Omega$. Suppose that $\operatorname{det} \nabla \mathbf{u} \in L_{\mathrm{loc}}^{1}(\Omega)$ and $\operatorname{cof} \nabla \mathbf{u} \in L_{\mathrm{loc}}^{1}\left(\Omega, \mathbb{R}^{n \times n}\right)$. For every $\mathbf{f} \in C_{c}^{\infty}\left(\Omega \times \mathbb{R}^{n}, \mathbb{R}^{n}\right)$, define

$$
\mathcal{E}_{\mathbf{u}}(\mathbf{f}):=\int_{\Omega}\left[\operatorname{cof} \nabla \mathbf{u}(\mathbf{x}) \cdot D_{\mathbf{x}} \mathbf{f}(\mathbf{x}, \mathbf{u}(\mathbf{x}))+\operatorname{det} \nabla \mathbf{u}(\mathbf{x}) \operatorname{div}_{\mathbf{y}} \mathbf{f}(\mathbf{x}, \mathbf{u}(\mathbf{x}))\right] \mathrm{d} \mathbf{x}
$$

and

$$
\mathcal{E}(\mathbf{u}):=\sup \left\{\mathcal{E}_{\mathbf{u}}(\mathbf{f}): \mathbf{f} \in C_{c}^{\infty}\left(\Omega \times \mathbb{R}^{n}, \mathbb{R}^{n}\right),\|\mathbf{f}\|_{\infty} \leq 1\right\} .
$$

Naturally, the notation $D_{\mathbf{x}} \mathbf{f}(\mathbf{x}, \mathbf{y})$ refers to the derivative of the map $\mathbf{f}(\cdot, \mathbf{y})$ evaluated at $\mathbf{x}$, while $\operatorname{div}_{\mathbf{y}} \mathbf{f}(\mathbf{x}, \mathbf{y})$ denotes the divergence of the map $\mathbf{f}(\mathbf{x}, \cdot)$ evaluated at y.

In order to motivate further Definition 2, suppose that $\mathbf{u}$ is as in Proposition 4. Then, the same proof that led to (9) also yields

$$
\mathcal{E}_{\mathbf{u}}(\mathbf{f})=\sum_{i=1}^{M} \int_{\Gamma_{i}} \mathbf{f}\left(\mathbf{u}^{-1}(\mathbf{y}), \mathbf{y}\right) \cdot \boldsymbol{\nu}(\mathbf{y}) \mathrm{d} \mathcal{H}^{n-1}(\mathbf{y}) .
$$

Analogously, for the deformations $\mathbf{u}_{j}$ depicted in Figure 3(b), the new energy functional is given by

$$
\mathcal{E}_{\mathbf{u}_{j}}(\mathbf{f})=\sum_{\mathbf{z} \in A_{j}^{-}} \int_{C_{j, \mathbf{z}}^{-}} \mathbf{f}(\mathbf{z}, \mathbf{y}) \cdot \boldsymbol{\nu}^{-}(\mathbf{y}) \mathrm{d} \mathcal{H}^{n-1}(\mathbf{y})+\sum_{\mathbf{z} \in A_{j}^{+}} \int_{C_{j, \mathbf{z}}^{+}} \mathbf{f}(\mathbf{z}, \mathbf{y}) \cdot \boldsymbol{\nu}^{+}(\mathbf{y}) \mathrm{d} \mathcal{H}^{n-1}(\mathbf{y}),
$$

which is the counterpart of (13).

The functional $\mathcal{E}$ is related to the identities

$$
\begin{aligned}
& \left(\operatorname{div}\left(\mathbf{f} \circ\left(\mathbf{u}^{-1} \bowtie \mathbf{i d}\right)\right)\right)(\mathbf{u}(\mathbf{x})) \operatorname{det} D \mathbf{u}(\mathbf{x}) \\
= & D_{\mathbf{x}} \mathbf{f}(\mathbf{x}, \mathbf{u}(\mathbf{x})) \cdot \operatorname{cof} D \mathbf{u}(\mathbf{x})+\left(\operatorname{div}_{\mathbf{y}} \mathbf{f}\right)(\mathbf{x}, \mathbf{u}(\mathbf{x})) \operatorname{det} D \mathbf{u}(\mathbf{x}) \\
= & \operatorname{Div}((\operatorname{adj} D \mathbf{u}(\mathbf{x})) \mathbf{f}(\mathbf{x}, \mathbf{u}(\mathbf{x})))
\end{aligned}
$$


in the same way that $\overline{\mathcal{E}}$ is related to (21). Here, $\mathbf{u}^{-1} \bowtie$ id is the function defined by $\left(\mathbf{u}^{-1} \bowtie \mathbf{i d}\right)(\mathbf{y}):=\left(\mathbf{u}^{-1}(\mathbf{y}), \mathbf{y}\right)$.

Finally, we mention that $\mathcal{E}_{\mathbf{u}}$ corresponds naturally to the $n-1$ vertical part of the boundary of the current $G_{\mathbf{u}}$, which is denoted by $\left(\partial G_{\mathbf{u}}\right)_{(n-1)}$. In particular our surface energy $\mathcal{E}(\mathbf{u})$ coincides with $\mathbb{M}\left(\left(\partial G_{\mathbf{u}}\right)_{(n-1)}\right)$.

As for the relation between $\overline{\mathcal{E}}$ and $\mathcal{E}$, we clearly have that $\overline{\mathcal{E}} \leq \mathcal{E}$. In addition, since the linear space spanned by the functions of the form $\Omega \times \mathbb{R}^{n} \ni(\mathbf{x}, \mathbf{y}) \mapsto \phi(\mathbf{x}) \mathbf{g}(\mathbf{y})$ with $\phi \in C_{c}^{\infty}(\Omega)$ and $\mathbf{g} \in C_{c}^{\infty}\left(\mathbb{R}^{n}, \mathbb{R}^{n}\right)$ is dense in $C_{c}^{\infty}\left(\Omega \times \mathbb{R}^{n}, \mathbb{R}^{n}\right)$ in the $C^{1}$ topology, it is easy to show that $\mathcal{E}(\mathbf{u})=0$ whenever $\overline{\mathcal{E}}(\mathbf{u})=0$. In particular, Propositions 2 and 3 provide sufficient conditions for the vanishing of $\mathcal{E}$, which generalize that of $[28, \mathrm{Rk}$. 3.2.3.3].

In spite of the fact that the boundedness of $\overline{\mathcal{E}}$ alone allows us to obtain the important results of Theorem 2 , it has been necessary to introduce the functional $\mathcal{E}$ for a number of reasons. In the first place, it is possible to construct examples (see Section 6) in which the energy $\overline{\mathcal{E}}$ does not give the area of the created surface, as one would desire. On the other hand, we do not know whether the boundedness of $\overline{\mathcal{E}}$ alone implies the full continuity of the determinant of the deformation gradient, that is, whether we can complement Theorem 2 with a result showing that the positive sign of the determinants of the gradients in a weakly converging sequence remains positive in the limit. In contrast, the boundedness of $\mathcal{E}$ does yield the weak continuity of the determinant, and we present it in Theorem 3 below. In addition, it can be proved (see [30]) that $\mathcal{E}(\mathbf{u})$ corresponds to the area of the created surface, as expected.

Before stating the above mentioned result, we write a few words on the relation between our theorem and previous results on this problem. Our proof follows that of [28, Th. 3.3.2.2], which in turn is based on [32]. In that paper, Müller [32] gave a simpler proof of a result by Giaquinta, Modica and Souček [27], which states that, in the Sobolev case, the vanishing of the boundary of the current (which implies the vanishing of $\mathcal{E}(\mathbf{u})$ ) implies the weak continuity of all minors. The original proof was based on the Federer-Fleming [20] closure theorem, whereas Müller's more direct proof followed a blow-up argument. In [28, Th. 3.3.2.2] Giaquinta, Modica and Souček noted that both Müller's proof and their original proof could be extended to the case when the mass of the boundary of the current is bounded (not necessarily zero).

In our Theorem 3 we show that if the cofactors are weakly continuous then it is not necessary to control the whole of the boundary of the graph of $\mathbf{u}$, but rather it suffices to control its $n-1$ vertical part. Since for this purpose we cannot assume that the mass of $\partial G_{\mathbf{u}}$ is bounded, we follow the extension by Giaquinta, Modica and Souček of the simpler proof by Müller using a blow-up argument.

Theorem 3 For each $j \in \mathbb{N}$, let $\mathbf{u}_{j}, \mathbf{u}: \Omega \rightarrow \mathbb{R}^{n}$ be measurable functions that are approximately differentiable in almost all $\Omega$,

$$
\operatorname{cof} \nabla \mathbf{u}_{j}, \operatorname{cof} \nabla \mathbf{u} \in L^{1}\left(\Omega, \mathbb{R}^{n \times n}\right), \quad \operatorname{det} \nabla \mathbf{u}_{j} \in L^{1}(\Omega) .
$$

Suppose that there exists $\theta \in L^{1}(\Omega)$ such that

$\mathbf{u}_{j} \rightarrow \mathbf{u}$ a.e., $\quad \operatorname{cof} \nabla \mathbf{u}_{j} \rightarrow \operatorname{cof} \nabla \mathbf{u} \quad$ in $L^{1}\left(\Omega, \mathbb{R}^{n \times n}\right), \quad \operatorname{det} \nabla \mathbf{u}_{j} \rightarrow \theta$ in $L^{1}(\Omega)$

as $j \rightarrow \infty$. Assume that

$$
\sup _{j \in \mathbb{N}} \mathcal{E}\left(\mathbf{u}_{j}\right)<\infty
$$


Then $\theta=\operatorname{det} \nabla \mathbf{u}$ a.e. and

$$
\mathcal{E}(\mathbf{u}) \leq \liminf _{j \rightarrow \infty} \mathcal{E}\left(\mathbf{u}_{j}\right)
$$

Proof Let $\mathbf{f} \in C_{c}^{\infty}\left(\Omega \times \mathbb{R}^{n}, \mathbb{R}^{n}\right)$ satisfy $\|\mathbf{f}\|_{\infty} \leq 1$. A standard convergence result (see, e.g., [40, Lemma 6.7]) yields

$$
\lim _{j \rightarrow \infty} \mathcal{E}_{\mathbf{u}_{j}}(\mathbf{f})=\int_{\Omega}\left[\operatorname{cof} \nabla \mathbf{u}(\mathbf{x}) \cdot D_{\mathbf{x}} \mathbf{f}(\mathbf{x}, \mathbf{u}(\mathbf{x}))+\theta(\mathbf{x}) \operatorname{div}_{\mathbf{y}} \mathbf{f}(\mathbf{x}, \mathbf{u}(\mathbf{x}))\right] \mathrm{d} \mathbf{x} .
$$

Since $\mathcal{E}_{\mathbf{u}_{j}}(\mathbf{f}) \leq \mathcal{E}\left(\mathbf{u}_{j}\right)$ for each $j \in \mathbb{N}$, thanks to Riesz' representation theorem, we obtain that the linear functional $\Lambda: C_{c}^{\infty}\left(\Omega \times \mathbb{R}^{n}, \mathbb{R}^{n}\right) \rightarrow \mathbb{R}$ given by

$$
\Lambda(\mathbf{f}):=\int_{\Omega}\left[\operatorname{cof} \nabla \mathbf{u}(\mathbf{x}) \cdot D_{\mathbf{x}} \mathbf{f}(\mathbf{x}, \mathbf{u}(\mathbf{x}))+\theta(\mathbf{x}) \operatorname{div}_{\mathbf{y}} \mathbf{f}(\mathbf{x}, \mathbf{u}(\mathbf{x}))\right] \mathrm{d} \mathbf{x}
$$

can be identified with an $\mathbb{R}^{n}$-valued measure in $\Omega \times \mathbb{R}^{n}$, and $|\Lambda|\left(\Omega \times \mathbb{R}^{n}\right) \leq \liminf _{j \rightarrow \infty} \mathcal{E}\left(\mathbf{u}_{j}\right)$. Here, $|\Lambda|$ denotes the total variation of the $\Lambda$, and is of course a measure too.

Now we claim that a.e. $\mathbf{x}_{0} \in \Omega$ satisfies

$$
\begin{aligned}
& \operatorname{cof} \nabla \mathbf{u}\left(\mathbf{x}_{0}+\varepsilon \mathbf{z}\right) \rightarrow \operatorname{cof} \nabla \mathbf{u}\left(\mathbf{x}_{0}\right) \quad \text { in } L_{\mathrm{loc}}^{1}\left(\mathbb{R}^{n}, \mathbb{R}^{n \times n}\right), \\
& \theta\left(\mathbf{x}_{0}+\varepsilon \mathbf{z}\right) \rightarrow \theta\left(\mathbf{x}_{0}\right) \quad \text { in } L_{\mathrm{loc}}^{1}\left(\mathbb{R}^{n}\right), \\
& \frac{\mathbf{u}\left(\mathbf{x}_{0}+\varepsilon \mathbf{z}\right)-\mathbf{u}\left(\mathbf{x}_{0}\right)}{\varepsilon} \rightarrow \nabla \mathbf{u}\left(\mathbf{x}_{0}\right) \mathbf{z} \text { in } L_{\mathrm{loc}}^{1}\left(\mathbb{R}^{n}, \mathbb{R}^{n}\right)
\end{aligned}
$$

as $\varepsilon \rightarrow 0$ (where of course $\mathbf{z} \in \mathbb{R}^{n}$ denotes the independent variable), and

$$
\limsup _{r \searrow 0} \frac{|\Lambda|\left(B\left(\left(\mathbf{x}_{0}, \mathbf{u}\left(\mathbf{x}_{0}\right)\right), r\right)\right)}{r^{n}}<\infty .
$$

Indeed, (25) is satisfied for any $\mathbf{x}_{0}$ that is a Lebesgue point of $\operatorname{cof} \nabla \mathbf{u}$ and of $\theta$, and a point of approximate differentiability of $\mathbf{u}$. To show (26) we use that, as $\Lambda$ is a Radon measure, the set

$$
\left\{(\mathbf{x}, \mathbf{y}) \in \Omega \times \mathbb{R}^{n}: \limsup _{r \searrow 0} \frac{|\Lambda|(B((\mathbf{x}, \mathbf{y}), r))}{r^{n}}=\infty\right\}
$$

has zero $\mathcal{H}^{n}$-measure (see for example [5, Th. 2.56]). In particular, the set

$$
\left\{(\mathbf{x}, \mathbf{u}(\mathbf{x})) \in \Omega_{d} \times \mathbb{R}^{n}: \limsup _{r \searrow 0} \frac{|\Lambda|(B((\mathbf{x}, \mathbf{u}(\mathbf{x})), r))}{r^{n}}=\infty\right\}
$$

and its orthogonal projection onto $\Omega_{d}$ are $\mathcal{H}^{n}$-null sets, where $\Omega_{d}$ is the set of approximate differentiability of $\mathbf{u}$. Hence (26) holds a.e. $\mathbf{x}_{0}$. We fix, until the end of the proof, an $\mathbf{x}_{0} \in \Omega_{d}$ such that (25) and (26). We shall prove that $\theta\left(\mathbf{x}_{0}\right)=\operatorname{det} \nabla \mathbf{u}\left(\mathbf{x}_{0}\right)$.

Given any $\mathbf{f} \in C_{c}^{\infty}\left(\mathbb{R}^{n} \times \mathbb{R}^{n}, \mathbb{R}^{n}\right)$ and $\varepsilon>0$ small enough, the set $\frac{\Omega-\mathbf{x}_{0}}{\varepsilon} \times \mathbb{R}^{n}$ contains spt $\mathbf{f}$. Hence, the function $\mathbf{f}_{\varepsilon}: \Omega \times \mathbb{R}^{n} \rightarrow \mathbb{R}^{n}$ defined by

$$
\mathbf{f}_{\varepsilon}(\mathbf{x}, \mathbf{y}):=\mathbf{f}\left(\frac{\mathbf{x}-\mathbf{x}_{0}}{\varepsilon}, \frac{\mathbf{y}-\mathbf{u}\left(\mathbf{x}_{0}\right)}{\varepsilon}\right), \quad(\mathbf{x}, \mathbf{y}) \in \Omega \times \mathbb{R}^{n}
$$


is compactly supported in $\Omega \times \mathbb{R}^{n}$. Then

$$
\begin{aligned}
\Lambda\left(\mathbf{f}_{\varepsilon}\right)=\varepsilon^{-1} \int_{\Omega}[ & \operatorname{cof} \nabla \mathbf{u}(\mathbf{x}) \cdot D_{\mathbf{x}} \mathbf{f}\left(\frac{\mathbf{x}-\mathbf{x}_{0}}{\varepsilon}, \frac{\mathbf{u}(\mathbf{x})-\mathbf{u}\left(\mathbf{x}_{0}\right)}{\varepsilon}\right) \\
+ & \left.\theta(\mathbf{x}) \operatorname{div} \mathbf{y} \mathbf{f}\left(\frac{\mathbf{x}-\mathbf{x}_{0}}{\varepsilon}, \frac{\mathbf{u}(\mathbf{x})-\mathbf{u}\left(\mathbf{x}_{0}\right)}{\varepsilon}\right)\right] \mathrm{d} \mathbf{x} \\
=\varepsilon^{n-1} \int_{\frac{\Omega-\mathbf{x}_{0}}{\varepsilon}} & {\left[\operatorname{cof} \nabla \mathbf{u}\left(\mathbf{x}_{0}+\varepsilon \mathbf{z}\right) \cdot D_{\mathbf{x}} \mathbf{f}\left(\mathbf{z}, \frac{\mathbf{u}\left(\mathbf{x}_{0}+\varepsilon \mathbf{z}\right)-\mathbf{u}\left(\mathbf{x}_{0}\right)}{\varepsilon}\right)\right.} \\
& \left.+\theta\left(\mathbf{x}_{0}+\varepsilon \mathbf{z}\right) \operatorname{div} \mathbf{y} \mathbf{f}\left(\mathbf{z}, \frac{\mathbf{u}\left(\mathbf{x}_{0}+\varepsilon \mathbf{z}\right)-\mathbf{u}\left(\mathbf{x}_{0}\right)}{\varepsilon}\right)\right] \mathrm{d} \mathbf{z} .
\end{aligned}
$$

Therefore, first extracting sequences $\varepsilon_{k} \searrow 0$, and then observing that the expression in the limit is independent of the sequence extracted, we may pass to the limit (see, e.g., [40, Lemma 6.7] for the details, if necessary) and obtain

$$
\lim _{\varepsilon \rightarrow 0} \frac{\Lambda\left(\mathbf{f}_{\varepsilon}\right)}{\varepsilon^{n-1}}=\int_{\mathbb{R}^{n}}\left[\operatorname{cof} \nabla \mathbf{u}\left(\mathbf{x}_{0}\right) \cdot D_{\mathbf{x}} \mathbf{f}\left(\mathbf{z}, \nabla \mathbf{u}\left(\mathbf{x}_{0}\right) \mathbf{z}\right)+\theta\left(\mathbf{x}_{0}\right) \operatorname{div} \mathbf{y} \mathbf{f}\left(\mathbf{z}, \nabla \mathbf{u}\left(\mathbf{x}_{0}\right) \mathbf{z}\right)\right] \mathrm{d} \mathbf{z} .
$$

Now observe that the limit function $\mathbb{R}^{n} \ni \mathbf{z} \mapsto \nabla \mathbf{u}\left(\mathbf{x}_{0}\right) \mathbf{z}$ is linear (hence regular), so, as explained at the beginning of this section,

$$
\int_{\mathbb{R}^{n}}\left[\operatorname{cof} \nabla \mathbf{u}\left(\mathbf{x}_{0}\right) \cdot \nabla_{\mathbf{x}} \mathbf{f}\left(\mathbf{z}, \nabla \mathbf{u}\left(\mathbf{x}_{0}\right) \mathbf{z}\right)+\operatorname{det} \nabla \mathbf{u}\left(\mathbf{x}_{0}\right) \operatorname{div} \mathbf{y} \mathbf{f}\left(\mathbf{z}, \nabla \mathbf{u}\left(\mathbf{x}_{0}\right) \mathbf{z}\right)\right] \mathrm{d} \mathbf{z}=0 .
$$

Therefore

$$
\lim _{\varepsilon \rightarrow 0} \frac{\Lambda\left(\mathbf{f}_{\varepsilon}\right)}{\varepsilon^{n-1}}=\left(\theta\left(\mathbf{x}_{0}\right)-\operatorname{det} \nabla \mathbf{u}\left(\mathbf{x}_{0}\right)\right) \int_{\mathbb{R}^{n}} \operatorname{div}_{\mathbf{y}} \mathbf{f}\left(\mathbf{z}, \nabla \mathbf{u}\left(\mathbf{x}_{0}\right) \mathbf{z}\right) \mathrm{d} \mathbf{z} .
$$

Thus, in order to show that $\theta\left(\mathbf{x}_{0}\right)=\operatorname{det} \nabla \mathbf{u}\left(\mathbf{x}_{0}\right)$, it suffices to prove that

$$
\lim _{\varepsilon \rightarrow 0} \frac{\Lambda\left(\mathbf{f}_{\varepsilon}\right)}{\varepsilon^{n-1}}=0 \quad \text { for every } \mathbf{f} \in C_{c}^{\infty}\left(\mathbb{R}^{n} \times \mathbb{R}^{n}, \mathbb{R}^{n}\right) .
$$

Indeed assuming (28) to be true, we choose $\mathbf{g} \in C_{c}^{\infty}\left(\mathbb{R}^{n}, \mathbb{R}^{n}\right)$ such that div $\mathbf{g}$ is not identically zero on $\nabla \mathbf{u}\left(\mathbf{x}_{0}\right)\left(\mathbb{R}^{n}\right)$, and define $\mathbf{f}: \mathbb{R}^{n} \times \mathbb{R}^{n} \rightarrow \mathbb{R}^{n}$ as

$$
\mathbf{f}(\mathbf{x}, \mathbf{y}):=\operatorname{div} \mathbf{g}\left(\nabla \mathbf{u}\left(\mathbf{x}_{0}\right) \mathbf{x}\right) \mathbf{g}(\mathbf{y}), \quad(\mathbf{x}, \mathbf{y}) \in \mathbb{R}^{n} \times \mathbb{R}^{n} ;
$$

then

$$
\int_{\mathbb{R}^{n}} \operatorname{div}_{\mathbf{y}} \mathbf{f}\left(\mathbf{z}, \nabla \mathbf{u}\left(\mathbf{x}_{0}\right) \mathbf{z}\right) \mathrm{d} \mathbf{z}=\int_{\mathbb{R}^{n}}(\operatorname{div} \mathbf{g})^{2}\left(\nabla \mathbf{u}\left(\mathbf{x}_{0}\right) \mathbf{z}\right) \mathrm{d} \mathbf{z} \neq 0
$$

and so $\theta\left(\mathbf{x}_{0}\right)=\operatorname{det} \nabla \mathbf{u}\left(\mathbf{x}_{0}\right)$.

In order to prove (28), we exploit the fact that $\Lambda$ is a measure in $\Omega \times \mathbb{R}^{n}$. Thus, for any $\mathbf{f} \in C_{c}^{\infty}\left(\mathbb{R}^{n} \times \mathbb{R}^{n}, \mathbb{R}^{n}\right)$ we can write

$$
\Lambda\left(\mathbf{f}_{\varepsilon}\right)=\int_{\Omega \times \mathbb{R}^{n}} \mathbf{f}_{\varepsilon}(\mathbf{x}, \mathbf{y}) \mathrm{d} \Lambda(\mathbf{x}, \mathbf{y}) .
$$

Let $R>0$ satisfy spt $\mathbf{f} \subset B((\mathbf{0}, \mathbf{0}), R)$; then $\operatorname{spt} \mathbf{f}_{\varepsilon} \subset B\left(\left(\mathbf{x}_{0}, \mathbf{u}\left(\mathbf{x}_{0}\right)\right), \varepsilon R\right)$ and

$$
\left|\Lambda\left(\mathbf{f}_{\varepsilon}\right)\right| \leq\|f\|_{\infty}|\Lambda|\left(B\left(\left(\mathbf{x}_{0}, \mathbf{u}\left(\mathbf{x}_{0}\right)\right), \varepsilon R\right)\right) .
$$

Therefore

$$
\frac{\left|\Lambda\left(\mathbf{f}_{\varepsilon}\right)\right|}{\varepsilon^{n-1}} \leq \varepsilon R^{n}\|f\|_{\infty} \frac{|\Lambda|\left(B\left(\left(\mathbf{x}_{0}, \mathbf{u}\left(\mathbf{x}_{0}\right)\right), \varepsilon R\right)\right.}{(\varepsilon R)^{n}},
$$

and (28) is obtained as a consequence of (26). 
Related to Theorems 2 and 3 is the following result, where we show that the weak continuity of the cofactor is implied by that of the determinant, provided that the energy $\mathcal{E}$ of the sequence is bounded.

Proposition $\mathbf{5}$ For each $j \in \mathbb{N}$, let $\mathbf{u}_{j}, \mathbf{u}: \Omega \rightarrow \mathbb{R}^{n}$ be measurable functions that are approximately differentiable in almost all $\Omega$, and

$$
\operatorname{cof} \nabla \mathbf{u}_{j} \in L^{1}\left(\Omega, \mathbb{R}^{n \times n}\right), \quad \operatorname{det} \nabla \mathbf{u}_{j}, \operatorname{det} \nabla \mathbf{u} \in L^{1}(\Omega) .
$$

Suppose that there exists $\vartheta \in L^{1}\left(\Omega, \mathbb{R}^{n \times n}\right)$ such that

$\mathbf{u}_{j} \rightarrow \mathbf{u}$ a.e., $\quad \operatorname{cof} \nabla \mathbf{u}_{j} \rightarrow \boldsymbol{\vartheta} \quad$ in $L^{1}\left(\Omega, \mathbb{R}^{n \times n}\right), \quad \operatorname{det} \nabla \mathbf{u}_{j} \rightarrow|\operatorname{det} \nabla \mathbf{u}| \quad$ in $L^{1}(\Omega)$ as $j \rightarrow \infty$. Assume that $\operatorname{det} \nabla \mathbf{u}(\mathbf{x}) \neq 0$ for a.e. $\mathbf{x} \in \Omega$, and that (23) holds. Then

$$
\vartheta=(\operatorname{sgn} \operatorname{det} \nabla \mathbf{u}) \operatorname{cof} \nabla \mathbf{u} \text { a.e. }
$$

Proof The same argument of the proof of Theorem 3 shows that for a.e. $\mathbf{x}_{0} \in \Omega$ and every $\mathbf{f} \in C_{c}^{\infty}\left(\mathbb{R}^{n} \times \mathbb{R}^{n}, \mathbb{R}^{n}\right)$,

$$
\int_{\mathbb{R}^{n}}\left[\boldsymbol{\vartheta}\left(\mathbf{x}_{0}\right) \cdot D_{\mathbf{x}} \mathbf{f}\left(\mathbf{z}, \nabla \mathbf{u}\left(\mathbf{x}_{0}\right) \mathbf{z}\right)+\left|\operatorname{det} \nabla \mathbf{u}\left(\mathbf{x}_{0}\right)\right| \operatorname{div}_{\mathbf{y}} \mathbf{f}\left(\mathbf{z}, \nabla \mathbf{u}\left(\mathbf{x}_{0}\right) \mathbf{z}\right)\right] \mathrm{d} \mathbf{z}=0
$$

As (27) holds too,

$$
\left(\boldsymbol{\vartheta}\left(\mathbf{x}_{0}\right)-\left(\operatorname{sgn} \operatorname{det} \nabla \mathbf{u}\left(\mathbf{x}_{0}\right)\right) \operatorname{cof} \nabla \mathbf{u}\left(\mathbf{x}_{0}\right)\right) \cdot \int_{\mathbb{R}^{n}} D_{\mathbf{x}} \mathbf{f}\left(\mathbf{z}, \nabla \mathbf{u}\left(\mathbf{x}_{0}\right) \mathbf{z}\right) \mathrm{d} \mathbf{z}=0 .
$$

Fix an $\mathbf{x}_{0} \in \Omega$ such that (30) holds and $\operatorname{det} \nabla \mathbf{u}\left(\mathbf{x}_{0}\right) \neq 0$. The proof will be finished as soon as we show that the set

$$
\left\{\int_{\mathbb{R}^{n}} D_{\mathbf{x}} \mathbf{f}\left(\mathbf{z}, \nabla \mathbf{u}\left(\mathbf{x}_{0}\right) \mathbf{z}\right) \mathrm{d} \mathbf{z}: \mathbf{f} \in C_{c}^{\infty}\left(\mathbb{R}^{n} \times \mathbb{R}^{n}, \mathbb{R}^{n}\right)\right\}
$$

contains a basis of $\mathbb{R}^{n \times n}$. Taking functions $\mathbf{f}$ of the form $\phi(\mathbf{x}) \mathbf{g}(\mathbf{y})$ with $\phi \in C_{c}^{\infty}\left(\mathbb{R}^{n}\right)$ and $\mathbf{g} \in C_{c}^{\infty}\left(\mathbb{R}^{n}, \mathbb{R}^{n}\right)$, and arguing with the coordinates of $\mathbf{g}$, it suffices to show that the set

$$
\left\{\int_{\mathbb{R}^{n}} D \phi(\mathbf{z}) g\left(\nabla \mathbf{u}\left(\mathbf{x}_{0}\right) \mathbf{z}\right) \mathrm{d} \mathbf{z}: \phi \in C_{c}^{\infty}\left(\mathbb{R}^{n}\right), g \in C_{c}^{\infty}\left(\mathbb{R}^{n}\right)\right\}
$$

contains a basis of $\mathbb{R}^{n}$. Choose any $\phi \in C_{c}^{\infty}\left(\mathbb{R}^{n}\right)$ such that the partial derivatives

$$
\frac{\partial \phi}{\partial z_{1}}, \ldots, \frac{\partial \phi}{\partial z_{n}}
$$

are linearly independent. As $L^{2}\left(\mathbb{R}^{n}\right)$ is a Hilbert space, for each $j \in\{1, \ldots, n\}$ there exists $\tilde{h}_{j} \in L^{2}\left(\mathbb{R}^{n}\right)$ such that

$$
\left\langle\frac{\partial \phi}{\partial z_{i}}, \tilde{h}_{j}\right\rangle=\delta_{i j}, \quad j \in\{1, \ldots, n\},
$$

where $\delta_{i j}$ denotes Kronecker's delta, and $\langle\cdot, \cdot\rangle$ the duality product in $L^{2}\left(\mathbb{R}^{n}\right)$. By mollification, there exists $h_{j} \in C_{c}^{\infty}\left(\mathbb{R}^{n}\right)$ such that the vectors

$$
\left(\left\langle\frac{\partial \phi}{\partial z_{1}}, h_{1}\right\rangle, \ldots,\left\langle\frac{\partial \phi}{\partial z_{1}}, h_{n}\right\rangle\right), \ldots,\left(\left\langle\frac{\partial \phi}{\partial z_{n}}, h_{1}\right\rangle, \ldots,\left\langle\frac{\partial \phi}{\partial z_{n}}, h_{n}\right\rangle\right)
$$


are linearly independent. Finally, for each $j \in\{1, \ldots, n\}$ define the function $g_{j}:=$ $h_{j} \circ \nabla \mathbf{u}\left(\mathbf{x}_{0}\right)^{-1}$. We have then shown that the vectors

$$
\int_{\mathbb{R}^{n}} D \phi(\mathbf{z}) g_{1}\left(\nabla \mathbf{u}\left(\mathbf{x}_{0}\right) \mathbf{z}\right) \mathrm{d} \mathbf{z}, \ldots, \int_{\mathbb{R}^{n}} D \phi(\mathbf{z}) g_{n}\left(\nabla \mathbf{u}\left(\mathbf{x}_{0}\right) \mathbf{z}\right) \mathrm{d} \mathbf{z}
$$

form a basis of $\mathbb{R}^{n}$. This concludes the proof.

Theorem 2 and Proposition 5 yield the following corollary.

Corollary 1 For each $j \in \mathbb{N}$, let $\mathbf{u}_{j}, \mathbf{u}: \Omega \rightarrow \mathbb{R}^{n}$ be approximately differentiable in almost all $\Omega$, and assume that (29) holds. Suppose that there exist $\vartheta \in L^{1}\left(\Omega, \mathbb{R}^{n \times n}\right)$ and $\theta \in L^{1}(\Omega)$ such that $\theta>0$ a.e., and

$$
\mathbf{u}_{j} \rightarrow \mathbf{u} \text { a.e., } \quad \operatorname{cof} \nabla \mathbf{u}_{j} \rightarrow \boldsymbol{\vartheta} \quad \text { in } L^{1}\left(\Omega, \mathbb{R}^{n \times n}\right), \quad \operatorname{det} \nabla \mathbf{u}_{j} \rightarrow \theta \quad \text { in } L^{1}(\Omega)
$$

as $j \rightarrow \infty$. Assume that (23) holds, and that for each $j \in \mathbb{N}$, the function $\mathbf{u}_{j}$ is oneto-one a.e. with $\operatorname{det} \nabla \mathbf{u}_{j}>0$ a.e. Then $\mathbf{u}$ is one-to-one a.e., $\boldsymbol{\vartheta}=(\operatorname{sgn} \operatorname{det} \nabla \mathbf{u}) \operatorname{cof} \nabla \mathbf{u}$ a.e., and $\theta=|\operatorname{det} \nabla \mathbf{u}|$ a.e.

This corollary provides us with a result of a different nature to the classic results on weak continuity of the determinant. Indeed, in Ball's [6] theory and all its many refinements, the weak continuity of the determinant is proved by induction: from the minors of order 2 to the determinant. The passage from the minors of order $i$ to those of order $i+1$ uses the Piola identities. The theory of Giaquinta, Modica and Souček [28] (as well as our Theorem 3) can be then regarded as a generalization of that methodology: the key idea being that it is not necessary to have the exact Piola identities, but, rather, that a control on how they fail is enough to make the induction step work. In contrast, Corollary 1 shows that, using the idea of Müller and Spector [34], the continuity (up to a sign) first of the determinant and then of the cofactor can be proved just from a bound on the surface energy. We do not know whether the full continuity of the determinant can be proved with this method without using the topological condition (INV) of [34].

\section{Existence of minimizers}

Once we have established the weak continuity of the determinant (Theorem 3), and the property that the weak limit of a sequence of one-to-one a.e. maps is one-to-one a.e. (Theorem 2), proving existence of minimizers is then standard. In this section we prove the existence of those minimizers for functionals in the context of nonlinear elasticity with cavitation and, perhaps, fracture. In order to simplify the exposition, we let $n=3$.

The first result concerns a model in which the elastic energy and the surface energy $\mathcal{E}$ are taken into account.

Theorem 4 Let $p \geq 2$. Let $\Omega$ be a bounded open set of $\mathbb{R}^{3}$ with (strongly) Lipschitz boundary. Let $\Gamma_{D} \subset \partial \Omega$ be a 2-rectifiable set with $\mathcal{H}^{2}\left(\Gamma_{D}\right)>0$. Let $\mathbf{b}: \Gamma_{D} \rightarrow \mathbb{R}^{3}$ be a measurable map. Define

$$
\mathcal{A}:=\left\{\mathbf{u} \in W^{1, p}\left(\Omega, \mathbb{R}^{3}\right): \operatorname{det} D \mathbf{u}>0 \text { a.e., } \mathbf{u} \text { is one-to-one a.e., }\left.\mathbf{u}\right|_{\Gamma_{D}}=\mathbf{b}\right\},
$$

the equality on $\Gamma_{D}$ being in the sense of traces. Let $\mathcal{R}:=\mathbb{R}^{3 \times 3} \times \mathbb{R}^{3 \times 3} \times(0, \infty)$. Let the function $W: \Omega \times \mathbb{R}^{3} \times \mathbb{R}^{3 \times 3} \rightarrow \mathbb{R}$ satisfy the following properties: 
i) There exists a function $\Phi: \Omega \times \mathbb{R}^{3} \times \mathcal{R} \rightarrow \mathbb{R}$ such that for a.e. $\mathbf{x} \in \Omega$ and every $\mathbf{y} \in \mathbb{R}^{3}$, the function $\Phi(\mathbf{x}, \mathbf{y}, \cdot)$ is convex and

$W(\mathbf{x}, \mathbf{y}, \mathbf{F})=\Phi(\mathbf{x}, \mathbf{y},(\mathbf{F}, \operatorname{cof} \mathbf{F}, \operatorname{det} \mathbf{F})) \quad$ for all $\mathbf{F} \in \mathbb{R}^{3 \times 3}$ such that $\operatorname{det} \mathbf{F}>0$.

ii) $\Phi(\mathbf{x}, \cdot, \cdot): \mathbb{R}^{3} \times \mathcal{R} \rightarrow \mathbb{R}$ is continuous for a.e. $\mathbf{x} \in \Omega$, and $\Phi(\cdot, \mathbf{y}, \mathbf{D}): \Omega \rightarrow \mathbb{R}$ is measurable for every $(\mathbf{y}, \mathbf{D}) \in \mathbb{R}^{3} \times \mathcal{R}$.

iii) There exist $a \in L^{1}(\Omega)$, a constant $c>0$, an increasing function $h_{1}:(0, \infty) \rightarrow$ $[0, \infty)$ and a convex function $h_{2}:(0, \infty) \rightarrow \mathbb{R}$ such that

$$
\lim _{t \rightarrow \infty} \frac{h_{1}(t)}{t}=\lim _{t \rightarrow \infty} \frac{h_{2}(t)}{t}=\lim _{t \rightarrow 0^{+}} h_{2}(t)=\infty
$$

and

$$
W(\mathbf{x}, \mathbf{y}, \mathbf{F}) \geq a(\mathbf{x})+c|\mathbf{F}|^{p}+h_{1}(|\operatorname{cof} \mathbf{F}|)+h_{2}(\operatorname{det} \mathbf{F})
$$

for a.e. $\mathbf{x} \in \Omega$, all $\mathbf{y} \in \mathbb{R}^{3}$ and all $\mathbf{F} \in \mathbb{R}^{3 \times 3}$ with $\operatorname{det} \mathbf{F}>0$.

Assume that $\mathcal{A} \neq \varnothing$, and define $I: \mathcal{A} \rightarrow \mathbb{R} \cup\{\infty\}$ as

$$
I(\mathbf{u}):=\int_{\Omega} W(\mathbf{x}, \mathbf{u}(\mathbf{x}), D \mathbf{u}(\mathbf{x})) \mathrm{d} \mathbf{x}+\mathcal{E}(\mathbf{u}), \quad \mathbf{u} \in \mathcal{A} .
$$

Then there exists a minimizer of $I$ in $\mathcal{A}$.

Proof If $I$ is identically $+\infty$, the result is trivial. Assume otherwise and note that iii) implies that $I$ is bounded below.

Let $\left\{\mathbf{u}_{j}\right\}_{j \in \mathbb{N}}$ be a minimizing sequence for $I$ in $\mathcal{A}$. Assumption iii) implies that the sequence $\left\{D \mathbf{u}_{j}\right\}_{j \in \mathbb{N}}$ is bounded in $L^{p}\left(\Omega, \mathbb{R}^{3}\right)$, whereas $\left\{\operatorname{cof} D \mathbf{u}_{j}\right\}_{j \in \mathbb{N}}$ and $\left\{\operatorname{det} D \mathbf{u}_{j}\right\}_{j \in \mathbb{N}}$ are equiintegrable by De La Vallée Poussin criterion. Thus, by the boundary condition and the Poincaré inequality, we obtain that there exist $\mathbf{u} \in W^{1, p}\left(\Omega, \mathbb{R}^{3}\right)$, $\vartheta \in L^{1}\left(\Omega, \mathbb{R}^{3 \times 3}\right)$ and $\theta \in L^{1}(\Omega)$ such that, for a subsequence (not relabelled),

$$
\begin{aligned}
& \mathbf{u}_{j} \rightarrow \mathbf{u} \text { in } W^{1, p}\left(\Omega, \mathbb{R}^{3}\right), \quad \mathbf{u}_{j} \rightarrow \mathbf{u} \text { a.e., } \\
& \operatorname{cof} D \mathbf{u}_{j} \rightarrow \boldsymbol{\vartheta} \text { in } L^{1}\left(\Omega, \mathbb{R}^{3 \times 3}\right), \quad \operatorname{det} D \mathbf{u}_{j} \rightarrow \theta \text { in } L^{1}(\Omega) .
\end{aligned}
$$

as $j \rightarrow \infty$. Clearly, $\theta \geq 0$ a.e. If $\theta$ were zero in a set $A$ of positive measure, then we would have (for a subsequence) $\operatorname{det} D \mathbf{u}_{j} \rightarrow 0$ in $L^{1}(A)$ and a.e. in $A$; hence by assumption iii), we obtain $h_{2}\left(\operatorname{det} D \mathbf{u}_{j}\right) \rightarrow \infty$ a.e. in $A$, as $j \rightarrow \infty$. Again by assumption iii) and Fatou's lemma, we get $I\left(\mathbf{u}_{j}\right) \rightarrow \infty$ as $j \rightarrow \infty$, which is a contradiction. Therefore, $\theta>0$ a.e.

As cof $D \mathbf{u}_{j}$ converges weakly to $\vartheta$ in $L^{1}$, and $\sup _{j \in \mathbb{N}}\left\|D \mathbf{u}_{j}\right\|_{L^{2}}<\infty$, by a standard result on weak continuity of minors (see, e.g., Ball, Currie and Olver [9, Th. 4.11], or by Lemma 2 below), we obtain $\vartheta=\operatorname{cof} D \mathbf{u}$ a.e. Thus, by Theorem $3, \theta=\operatorname{det} D \mathbf{u}$ a.e. and (24). Theorem 2, on the other hand, shows that $\mathbf{u}$ is one-to-one a.e. Since the boundary condition is also preserved under the limit, we conclude that $\mathbf{u} \in \mathcal{A}$. We are then in a position to apply the lower semicontinuity theorem of [9, Th. 5.4], according to which

$$
\int_{\Omega} W(\mathbf{x}, \mathbf{u}(\mathbf{x}), D \mathbf{u}(\mathbf{x})) \mathrm{d} \mathbf{x} \leq \liminf _{j \rightarrow \infty} \int_{\Omega} W\left(\mathbf{x}, \mathbf{u}_{j}(\mathbf{x}), D \mathbf{u}_{j}(\mathbf{x})\right) \mathrm{d} \mathbf{x} .
$$

This inequality and (24) conclude that $I(\mathbf{u}) \leq \liminf _{j \rightarrow \infty} I\left(\mathbf{u}_{j}\right)$, and hence $\mathbf{u}$ is a minimizer of $I$ in $\mathcal{A}$. 
In Theorem 4, as we have explained, elastic and surface energy are taken into account. Fracture, in contrast, is not allowed, since Sobolev functions cannot have jump discontinuities over a 2-dimensional surface. To allow for fracture, the usual framework is a functional space modelled over $S B V$. Typical existence results are set in $S B V$ under an $L^{\infty}$ a priori bound on the deformations, or in $G S B V$ under an $L^{1}$ coercivity property of the energy; see, e.g., the approaches of $[2,4,23,26]$. In our formulation, we have chosen the $S B V$ setting with an $L^{\infty}$ a priori bound on the deformation. As for the Dirichlet conditions, we permit the possibility of disagreement with the boundary condition at the expense of a penalization in the energy (as in $[4,15]$ ).

The existence theory in $S B V$ requires, in addition, the following preliminary result on the weak continuity of the cofactor. This is the $S B V$ counterpart (essentially due to Ambrosio [3]) of a better-known result in the Sobolev setting. We will not provide a proof, since it is identical to that of [3, Cor. 4.9] (see also [5, Cor. 5.31]).

Lemma 2 For each $j \in \mathbb{N}$, let $\mathbf{u}_{j}, \mathbf{u} \in S B V\left(\Omega, \mathbb{R}^{3}\right)$ satisfy that the sequences $\left\{\left\|\nabla \mathbf{u}_{j}\right\|_{L^{2}}\right\}_{j \in \mathbb{N}}$ and $\left\{\mathcal{H}^{2}\left(J_{\mathbf{u}_{j}}\right)\right\}_{j \in \mathbb{N}}$ are bounded. Assume that $\mathbf{u}_{j} \rightarrow \mathbf{u}$ in $L^{1}\left(\Omega, \mathbb{R}^{3}\right)$ as $j \rightarrow \infty$, and the sequence $\left\{\left|\operatorname{cof} \nabla \mathbf{u}_{j}\right|\right\}_{j \in \mathbb{N}}$ is equiintegrable. Then

$$
\operatorname{cof} \nabla \mathbf{u}_{j} \rightarrow \operatorname{cof} \nabla \mathbf{u} \quad \text { in } L^{1}\left(\Omega, \mathbb{R}^{3 \times 3}\right) \quad \text { as } j \rightarrow \infty .
$$

We are then in a position to prove the second existence theorem of this paper. It concerns a model in which the elastic energy, the surface energy $\mathcal{E}$ and the fracture energy are taken into account.

Theorem 5 Let $p \geq 2$ and $\lambda_{1}, \lambda_{2}>0$. Let $\Omega$ be a bounded open set of $\mathbb{R}^{3}$ with (strongly) Lipschitz boundary. Let $\Gamma_{D} \subset \partial \Omega$ be a 2-rectifiable set, and let $K \subset \mathbb{R}^{3}$ be compact. Let $\Omega^{\prime}$ be an open set containing $\Omega \cup \Gamma_{D}$ such that $\mathcal{H}^{2}\left(\partial \Omega \backslash\left(\partial \Omega^{\prime} \cup \Gamma_{D}\right)\right)=0$. Let $\mathbf{b} \in S B V\left(\Omega^{\prime}, \mathbb{R}^{3}\right)$ satisfy $\mathbf{b}(\mathbf{x}) \in K$ for a.e. $\mathbf{x} \in \Omega^{\prime} \backslash \Omega$, and denote by $\mathbf{b}^{+}$the lateral trace of $\mathbf{b}$ on $\partial \Omega$ corresponding to $\Omega^{\prime} \backslash\left(\Omega \cup \Gamma_{D}\right)$. Assume that $\mathcal{H}^{2}\left(J_{\mathbf{b}} \cap \Omega^{\prime} \backslash \bar{\Omega}\right)<\infty$. Define $\mathcal{A}$ as the set of functions $\mathbf{u} \in S B V\left(\Omega, \mathbb{R}^{3}\right)$ such that

$\nabla \mathbf{u} \in L^{p}\left(\Omega, \mathbb{R}^{3 \times 3}\right), \quad \operatorname{det} \nabla \mathbf{u}>0$ a.e., $\quad \mathbf{u}$ is one-to-one a.e., $\quad \mathbf{u}(\mathbf{x}) \in K$ a.e. $\mathbf{x} \in \Omega$,

Let the function $W: \Omega \times \mathbb{R}^{3} \times \mathbb{R}^{3 \times 3} \rightarrow \mathbb{R}$ satisfy properties i)-iii) of Theorem 4 .

Assume that $\mathcal{A} \neq \varnothing$, and define $I: \mathcal{A} \rightarrow \mathbb{R} \cup\{\infty\}$, for each $\mathbf{u} \in \mathcal{A}$, as

$$
\begin{aligned}
I(\mathbf{u}):= & \int_{\Omega} W(\mathbf{x}, \mathbf{u}(\mathbf{x}), \nabla \mathbf{u}(\mathbf{x})) \mathrm{d} \mathbf{x} \\
& +\lambda_{1} \mathcal{E}(\mathbf{u})+\lambda_{2} \mathcal{H}^{2}\left(J_{\mathbf{u}}\right)+\lambda_{2} \mathcal{H}^{2}\left(\left\{\mathbf{x} \in \Gamma_{D}: \mathbf{b}^{+}(\mathbf{x}) \neq \mathbf{u}(\mathbf{x})\right\}\right)
\end{aligned}
$$

the value of $\mathbf{u}$ in $\Gamma_{D}$ being in the sense of traces. Then there exists a minimizer of $I$ in $\mathcal{A}$.

Proof If $I$ is identically $+\infty$, the result is trivial. Assume otherwise and note that iii) implies that $I$ is bounded below.

Let $\left\{\mathbf{u}_{j}\right\}_{j \in \mathbb{N}}$ be a minimizing sequence for $I$ in $\mathcal{A}$. Assumption iii) and the definitions of $I$ and $\mathcal{A}$ imply that

$$
\sup _{j \in \mathbb{N}}\left[\left\|\mathbf{u}_{j}\right\|_{L^{\infty}}+\left\|\nabla \mathbf{u}_{j}\right\|_{L^{p}}+\mathcal{H}^{2}\left(J_{\mathbf{u}_{j}}\right)\right]<\infty .
$$


Therefore, by the compactness theorem of Ambrosio [1, Prop. 4.3] (see also [5, Th. $4.8]$ ), there exists $\mathbf{u} \in S B V\left(\Omega, \mathbb{R}^{3}\right)$ such that, for a subsequence (not rellabelled),

$$
\mathbf{u}_{j} \rightarrow \mathbf{u} \text { in } L^{1}\left(\Omega, \mathbb{R}^{3}\right) \text { and a.e., } \quad \nabla \mathbf{u}_{j} \rightarrow \nabla \mathbf{u} \text { in } L^{p}\left(\Omega, \mathbb{R}^{3 \times 3}\right) \quad \text { as } j \rightarrow \infty .
$$

Moreover, $\mathcal{H}^{2}\left(J_{\mathbf{u}}\right) \leq \liminf _{j \rightarrow \infty} \mathcal{H}^{2}\left(J_{\mathbf{u}_{j}}\right)$ and $\mathbf{u}(\mathbf{x}) \in K$ a.e. $\mathbf{x} \in \Omega$. Now, for each $j \in$ $\mathbb{N}$, define the functions $\tilde{\mathbf{u}}_{j}, \tilde{\mathbf{u}}: \Omega^{\prime} \rightarrow \mathbb{R}^{3}$ as the extensions by $\mathbf{b}$ to $\Omega^{\prime} \backslash \Omega$ of $\mathbf{u}_{j}, \mathbf{u}$, respectively. Repeating the above argument, we obtain that $\mathcal{H}^{2}\left(J_{\tilde{\mathbf{u}}}\right) \leq \liminf _{j \rightarrow \infty} \mathcal{H}^{2}\left(J_{\tilde{\mathbf{u}}_{j}}\right)$. Now note that the assumptions on $\Omega, \Omega^{\prime}$ and $\Gamma_{D}$ imply that

$$
\mathcal{H}^{2}\left(J_{\tilde{\mathbf{u}}}\right)=\mathcal{H}^{2}\left(J_{\mathbf{u}}\right)+\mathcal{H}^{2}\left(J_{\mathbf{b}} \cap \Omega^{\prime} \backslash \bar{\Omega}\right)+\mathcal{H}^{2}\left(\left\{\mathbf{x} \in \Gamma_{D}: \mathbf{b}^{+}(\mathbf{x}) \neq \mathbf{u}(\mathbf{x})\right\}\right) .
$$

Since an analogous inequality holds for $\mathbf{u}_{j}$ (replacing $\mathbf{u}$ ), we conclude that

$$
\mathcal{H}^{2}\left(J_{\mathbf{u}}\right)+\mathcal{H}^{2}\left(\Gamma_{D} \cap\left\{\mathbf{b}^{+} \neq \mathbf{u}\right\}\right) \leq \liminf _{j \rightarrow \infty}\left[\mathcal{H}^{2}\left(J_{\mathbf{u}_{j}}\right)+\mathcal{H}^{2}\left(\Gamma_{D} \cap\left\{\mathbf{b}^{+} \neq \mathbf{u}_{j}\right\}\right)\right] .
$$

On the other hand, assumption iii) and De La Vallée Poussin criterion imply the existence of $\vartheta \in L^{1}\left(\Omega, \mathbb{R}^{3 \times 3}\right)$ and $\theta \in L^{1}(\Omega)$ such that, for a subsequence (not relabelled),

$$
\operatorname{cof} \nabla \mathbf{u}_{j} \rightarrow \boldsymbol{\vartheta} \text { in } L^{1}\left(\Omega, \mathbb{R}^{3 \times 3}\right), \quad \operatorname{det} \nabla \mathbf{u}_{j} \rightarrow \theta \text { in } L^{1}(\Omega) \quad \text { as } j \rightarrow \infty
$$

As in the proof of Theorem $4, \theta>0$ a.e. Now, Lemma 2 shows that $\vartheta=\operatorname{cof} \nabla \mathbf{u}$ a.e., Theorem 3 shows that $\theta=\operatorname{det} \nabla \mathbf{u}$ a.e. and (24), and Theorem 2 shows that $\mathbf{u}$ is oneto-one a.e. We thus conclude that $\mathbf{u} \in \mathcal{A}$. Now, by the lower semicontinuity theorem of [9, Th. 5.4],

$$
\int_{\Omega} W(\mathbf{x}, \mathbf{u}(\mathbf{x}), \nabla \mathbf{u}(\mathbf{x})) \mathrm{d} \mathbf{x} \leq \liminf _{j \rightarrow \infty} \int_{\Omega} W\left(\mathbf{x}, \mathbf{u}_{j}(\mathbf{x}), \nabla \mathbf{u}_{j}(\mathbf{x})\right) \mathrm{d} \mathbf{x}
$$

This inequality, together with (31) and (24), concludes that $I(\mathbf{u}) \leq \liminf _{j \rightarrow \infty} I\left(\mathbf{u}_{j}\right)$, and hence $\mathbf{u}$ is a minimizer of $I$ in $\mathcal{A}$.

We observe that the energy $I$ of Theorem 5 penalizes the formation of cracks both in the reference configuration (through $\mathcal{E}(\mathbf{u})$ ) and in the deformed configuration (through $\left.\mathcal{H}^{2}\left(J_{\mathbf{u}}\right)\right)$. A possible justification is that the energy $\mathcal{H}^{2}\left(J_{\mathbf{u}}\right)$ measures the breaking of atomic bonds, while $\mathcal{E}(\mathbf{u})$ accounts for the stretching of a fracture surface (the latter would be negligible, due to the assumption of small deformations, in the context of classical fracture mechanics). That both energies should be taken into account has been hypothesized by Gent and Wang [25]. In any case, Corollary 1 suggests that it might be possible to build an existence theory for cavitation and fracture without including the term $\mathcal{H}^{2}\left(J_{\mathbf{u}}\right)$. Of course, extra conditions guaranteeing the full continuity of the determinant (not only of its absolute value) and the compactness in $S B V$ are necessary.

As we said at the beginning of the section, Theorems 2 and 3, together with standard minimization techniques in nonlinear elasticity with or without fracture, provide results on existence of minimizers, of which Theorems 4 and 5 are two examples. In Section 7 we will make some comments on related models. 


\section{$6 \overline{\mathcal{E}}$ versus $\mathcal{E}$}

It is easy to check that $\overline{\mathcal{E}}(\mathbf{u})=\mathcal{E}(\mathbf{u})$ when $\mathbf{u}$ satisfies the assumptions of Proposition 4 , or when $\mathbf{u}$ equals any of the deformations $\mathbf{u}_{j}$ depicted in Figure 3(b). In this section we prove, by means of an example, that $\overline{\mathcal{E}}$ and $\mathcal{E}$ do not coincide, and, moreover, we show that $\overline{\mathcal{E}}(\mathbf{u})$ is not a physical quantity and exhibits a sensitive dependence on $\mathbf{u}$.

The constructions is as follows. Let $\Omega$ be the ball $B(\mathbf{0}, 2)$ in $\mathbb{R}^{2}$, let $0<\theta_{0}<2 \pi$, let $\mathbf{R} \in \mathbb{R}^{2 \times 2}$ be the rotation matrix corresponding to an angle of $\theta_{0}$, and consider the function $\mathbf{u}: \Omega \rightarrow \mathbb{R}^{2}$ defined as

$$
\mathbf{u}(\mathbf{x}):= \begin{cases}\mathbf{R x} & \text { if }|\mathbf{x}|<1 \\ \mathbf{x} & \text { if } 1 \leq|\mathbf{x}|<2 .\end{cases}
$$

First we show that $\mathcal{E}(\mathbf{u})=4 \pi$, and then we show that $\overline{\mathcal{E}}(\mathbf{u})$ has a sensitive dependence on the rotation angle $\theta_{0}$. Thus, in this example, $\mathcal{E}$ measures the (1-dimensional) 'area' of the created (1-dimensional) 'surface'. Indeed, the created surface is $\partial B(\mathbf{0}, 1)$, but counted twice: as the surface created in $B(\mathbf{0}, 1)$ plus the surface created in $\Omega \backslash \bar{B}(\mathbf{0}, 1)$. It may help the reader to consider the map $\tilde{\mathbf{u}}: \Omega \rightarrow \mathbb{R}^{2}$ defined as $\tilde{\mathbf{u}}(\mathbf{x})=\mathbf{R x}$ for $|\mathbf{x}|<1$, and $\tilde{\mathbf{u}}(\mathbf{x})=\mathbf{x}+\mathbf{a}$ for $1 \leq|\mathbf{x}|<2$ and some $\mathbf{a} \in \mathbb{R}^{2}$. It then becomes clearer why the surface $\partial B(\mathbf{0}, 1)$ created by $\mathbf{u}$ must be counted twice.

In order to calculate $\mathcal{E}(\mathbf{u})$ we take $\mathbf{f} \in C_{c}^{\infty}\left(\Omega \times \mathbb{R}^{2}, \mathbb{R}^{2}\right)$, and use the divergence theorem to obtain

$$
\begin{aligned}
\mathcal{E}_{\mathbf{u}}(\mathbf{f}) & =\left[\int_{B(\mathbf{0}, 1)}+\int_{B(\mathbf{0}, 2) \backslash \bar{B}(\mathbf{0}, 1)}\right] \operatorname{Div}[\operatorname{adj} \nabla \mathbf{u}(\mathbf{x}) \mathbf{f}(\mathbf{x}, \mathbf{u}(\mathbf{x}))] \mathrm{d} \mathbf{x} \\
& =\int_{\partial B(\mathbf{0}, 1)}\left[\mathbf{R}^{T} \mathbf{f}(\mathbf{x}, \mathbf{R} \mathbf{x})-\mathbf{f}(\mathbf{x}, \mathbf{x})\right] \cdot \mathbf{x} \mathrm{d} \mathcal{H}^{1}(\mathbf{x}) .
\end{aligned}
$$

Therefore, $\mathcal{E}(\mathbf{u}) \leq 4 \pi$. Considering now any $\overline{\mathbf{f}} \in C_{c}^{\infty}\left(\Omega \times \mathbb{R}^{2}, \mathbb{R}^{2}\right)$ such that $\|\overline{\mathbf{f}}\|_{\infty}=1$ and

$$
\overline{\mathbf{f}}(\mathbf{x}, \mathbf{x})=-\mathbf{x}, \quad \overline{\mathbf{f}}(\mathbf{x}, \mathbf{R} \mathbf{x})=\mathbf{R} \mathbf{x}, \quad \mathbf{x} \in \partial B(\mathbf{0}, 1)
$$

we conclude that

$$
\mathcal{E}(\mathbf{u}) \geq \mathcal{E}_{\mathbf{u}}(\overline{\mathbf{f}})=\int_{\partial B(\mathbf{0}, 1)} 2 \mathbf{x} \cdot \mathbf{x} \mathrm{d} \mathcal{H}^{1}(\mathbf{x})=4 \pi
$$

Therefore, $\mathcal{E}(\mathbf{u})=4 \pi$.

The sensitive dependence of $\overline{\mathcal{E}}(\mathbf{u})$ upon the rotation angle $\theta_{0}$ is described in the following proposition.

Proposition 6 Consider the function $\mathbf{u}$ defined in (32). Then the following statements are equivalent:

i) $\overline{\mathcal{E}}(\mathbf{u})=4 \pi$.

ii) For each $j \in \mathbb{N}$ there exist $\varepsilon_{j}>0$ and $a_{j}, b_{j} \in \mathbb{N}$ with $a_{j}$ odd such that

$$
\left|\theta_{0}-\frac{a_{j}}{b_{j}} \pi\right| \leq \varepsilon_{j} \quad \text { and } \quad \lim _{j \rightarrow \infty} \varepsilon_{j} b_{j}=0
$$

iii) $\theta_{0} / \pi$ is either irrational or of the form $\frac{a}{b}$ with $a$ odd and $b \in \mathbb{N}$. 
Proof First, we assume that ii) holds and show that i) holds.

For all $\phi \in C_{c}^{\infty}(\Omega)$ and $\mathbf{g} \in C_{c}^{\infty}\left(\mathbb{R}^{2}, \mathbb{R}^{2}\right)$, as in (33),

$$
\begin{aligned}
\overline{\mathcal{E}}_{\mathbf{u}}(\phi, \mathbf{g}) & =\int_{\partial B(\mathbf{0}, 1)}\left[\mathbf{R}^{T} \phi(\mathbf{x}) \mathbf{g}(\mathbf{R} \mathbf{x})-\phi(\mathbf{x}) \mathbf{g}(\mathbf{x})\right] \cdot \mathbf{x} \mathrm{d} \mathcal{H}^{1}(\mathbf{x}) \\
& =\int_{\partial B(\mathbf{0}, 1)}\left[\phi\left(\mathbf{R}^{T} \mathbf{x}\right)-\phi(\mathbf{x})\right] \mathbf{g}(\mathbf{x}) \cdot \mathbf{x} \mathrm{d} \mathcal{H}^{1}(\mathbf{x})
\end{aligned}
$$

For $j \in \mathbb{N}$ large enough, define the sets

$$
\begin{aligned}
& A_{j}:=\left\{(\cos \theta, \sin \theta): \theta \in \bigcup_{i=0}^{b_{j}-1}\left[\frac{2 i \pi+\varepsilon_{j}}{b_{j}}, \frac{(2 i+1) \pi-\varepsilon_{j}}{b_{j}}\right]\right\}, \\
& B_{j}:=\left\{(\cos \theta, \sin \theta): \theta \in \bigcup_{i=1}^{b_{j}}\left[\frac{(2 i-1) \pi+\varepsilon_{j}}{b_{j}}, \frac{2 i \pi-\varepsilon_{j}}{b_{j}}\right]\right\} .
\end{aligned}
$$

Define $\theta_{j}:=\frac{a_{j}}{b_{j}} \pi$, and denote by $\mathbf{R}_{j}$ the rotation matrix corresponding to an angle of $\theta_{j}$. Note that $\mathbf{R}_{j}^{T} A_{j}=B_{j}$ and $\mathbf{R}_{j}^{T} B_{j}=A_{j}$. In addition,

$$
\mathbf{R} A_{j}=\left\{(\cos \theta, \sin \theta): \theta \in \bigcup_{i=1}^{b_{j}}\left[\frac{(2 i-1) \pi+\varepsilon_{j}}{b_{j}}+\theta_{0}-\theta_{j}, \frac{2 i \pi-\varepsilon_{j}}{b_{j}}+\theta_{0}-\theta_{j}\right]\right\}
$$

and

$$
\mathbf{R} B_{j}=\left\{(\cos \theta, \sin \theta): \theta \in \bigcup_{i=0}^{b_{j}-1}\left[\frac{2 i \pi+\varepsilon_{j}}{b_{j}}+\theta_{0}-\theta_{j}, \frac{(2 i+1) \pi-\varepsilon_{j}}{b_{j}}+\theta_{0}-\theta_{j}\right]\right\} .
$$

Note that

$$
\mathcal{H}^{1}\left(A_{j} \cap \mathbf{R} B_{j}\right)=\mathcal{H}^{1}\left(\mathbf{R} A_{j} \cap B_{j}\right)=\pi-2 \varepsilon_{j}-b_{j}\left|\theta_{j}-\theta_{0}\right| .
$$

For each $j \in \mathbb{N}$ large enough, choose functions $\phi_{j} \in C_{c}^{\infty}(\Omega)$ and $\mathbf{g}_{j} \in C_{c}^{\infty}\left(\mathbb{R}^{2}, \mathbb{R}^{2}\right)$ such that $\left\|\phi_{j}\right\|_{\infty}=\left\|\mathbf{g}_{j}\right\|_{\infty}=1$ and

$$
\phi_{j}(\mathbf{x})=-1, \quad \mathbf{g}_{j}(\mathbf{x})=\mathbf{x} \quad \text { for } \quad \mathbf{x} \in A_{j} ; \quad \phi_{j}(\mathbf{x})=1, \quad \mathbf{g}_{j}(\mathbf{x})=-\mathbf{x} \quad \text { for } \quad \mathbf{x} \in B_{j} .
$$

Then, using (34) and (35), for $j$ large enough,

$$
\begin{aligned}
\overline{\mathcal{E}}_{\mathbf{u}}\left(\phi_{j}, \mathbf{g}_{j}\right) \geq & 2 \mathcal{H}^{1}\left(A_{j} \cap \mathbf{R} B_{j}\right)+2 \mathcal{H}^{1}\left(\mathbf{R} A_{j} \cap B_{j}\right) \\
& -2 \mathcal{H}^{1}\left(\partial B(\mathbf{0}, 1) \backslash\left[\left(A_{j} \cap \mathbf{R} B_{j}\right) \cup\left(\mathbf{R} A_{j} \cap B_{j}\right)\right]\right) \\
\geq & 4 \pi-16 \varepsilon_{j}-8 \varepsilon_{j} b_{j} .
\end{aligned}
$$

Taking limits as $j \rightarrow \infty$ we conclude that $\overline{\mathcal{E}}(\mathbf{u})=4 \pi$.

Now we prove that i) implies iii). Indeed, suppose, looking for a contradiction, that $\overline{\mathcal{E}}(\mathbf{u})=4 \pi$ and $\frac{\theta_{0}}{\pi}=\frac{a}{b}$ for some $a, b \in \mathbb{N}$ with $a$ even and $b$ odd. From (34), for every $\varepsilon>0$ there exists $\phi \in C_{c}^{\infty}(\Omega)$ with $\|\phi\|_{\infty} \leq 1$ such that

$$
\int_{\partial B(0,1)}\left|\phi\left(\mathbf{R}^{T} \mathbf{x}\right)-\phi(\mathbf{x})\right| \mathrm{d} \mathcal{H}^{1}(\mathbf{x}) \geq(4-\varepsilon) \pi .
$$


Since $\mathbf{R}^{b}=\mathbf{1}$ and $b$ is odd, from the triangular inequality we obtain that

$$
\begin{aligned}
\int_{\partial B(\mathbf{0}, 1)} \mid \phi\left(\mathbf{R}^{T} \mathbf{x}\right) & -\phi(\mathbf{x})\left|\mathrm{d} \mathcal{H}^{1}(\mathbf{x}) \leq 2 \int_{\partial B(\mathbf{0}, 1)}\right| \phi(\mathbf{x}) \mid \mathrm{d} \mathcal{H}^{1}(\mathbf{x}) \\
& =\int_{\partial B(\mathbf{0}, 1)}\left|\phi\left(\mathbf{R}^{b} \mathbf{x}\right)+\phi(\mathbf{x})\right| \mathrm{d} \mathcal{H}^{1}(\mathbf{x}) \\
& \leq \int_{\partial B(\mathbf{0}, 1)} \sum_{j=1}^{b}\left|(-1)^{j-1} \phi\left(\mathbf{R}^{j} \mathbf{x}\right)+(-1)^{j-1} \phi\left(\mathbf{R}^{j-1} \mathbf{x}\right)\right| \mathrm{d} \mathcal{H}^{1}(\mathbf{x}) \\
& =b \int_{\partial B(\mathbf{0}, 1)}\left|\phi\left(\mathbf{R}^{T} \mathbf{x}\right)+\phi(\mathbf{x})\right| \mathrm{d} \mathcal{H}^{1}(\mathbf{x}),
\end{aligned}
$$

where the last equality follows from a change of variables. Since

$$
\left|\phi\left(\mathbf{R}^{T} \mathbf{x}\right)-\phi(\mathbf{x})\right|+\left|\phi\left(\mathbf{R}^{T} \mathbf{x}\right)+\phi(\mathbf{x})\right|=2 \max \left\{\left|\phi\left(\mathbf{R}^{T} \mathbf{x}\right)\right|,|\phi(\mathbf{x})|\right\} \leq 2
$$

for $\mathbf{x} \in \partial B(\mathbf{0}, 1)$, it follows that

$$
\int_{\partial B(\mathbf{0}, 1)}\left|\phi\left(\mathbf{R}^{T} \mathbf{x}\right)+\phi(\mathbf{x})\right| \mathrm{d} \mathcal{H}^{1}(\mathbf{x}) \leq \varepsilon \pi .
$$

Altogether we have

$$
(4-\varepsilon) \pi \leq \int_{\partial B(\mathbf{0}, 1)}\left|\phi\left(\mathbf{R}^{T} \mathbf{x}\right)-\phi(\mathbf{x})\right| \mathrm{d} \mathcal{H}^{1}(\mathbf{x}) \leq 2 \int_{\partial B(\mathbf{0}, 1)}|\phi(\mathbf{x})| \mathrm{d} \mathcal{H}^{1}(\mathbf{x}) \leq b \varepsilon \pi .
$$

For $\varepsilon$ small enough this is a contradiction.

Finally, we show that iii) implies ii). The conclusion is trivial if $\theta_{0} / \pi$ is of the form $\frac{a}{b}$ with $a$ odd and $b \in \mathbb{N}$. Assume, then, that $\theta_{0} / \pi$ is irrational. The proof of this will use Farey fractions, which is a standard tool in rational approximation. Their definition and elementary properties can be found, e.g., in [36, Ch. 6].

For each $j \in \mathbb{N}$, there exist $p_{j}, q_{j}, r_{j}, s_{j} \in \mathbb{N}$ (uniquely determined) such that $\frac{p_{j}}{q_{j}}$ and $\frac{r_{j}}{s_{j}}$ are Farey fractions of order $j$, no other Farey fraction of order $j$ lies between them, and $\frac{p_{j}}{q_{j}}<\frac{\theta_{0}}{\pi}<\frac{r_{j}}{s_{j}}$. By [36, Th. 6.4], $q_{j} \leq q_{j+1}$ and $s_{j} \leq s_{j+1}$. Moreover, the sets $\left\{q_{i}: i \in \mathbb{N}\right\}$ and $\left\{s_{i}: i \in \mathbb{N}\right\}$ are infinite, since otherwise $\theta_{0} / \pi$ would be rational. It follows that $\lim _{i \rightarrow \infty} q_{i}=\lim _{i \rightarrow \infty} s_{i}=\infty$. By [36, Th. 6.1], one of the fractions $\frac{p_{j}}{q_{j}}$, $\frac{r_{j}}{s_{j}}$ has odd numerator; call $a_{j}$ its numerator, $b_{j}$ its denominator, and

$$
\varepsilon_{j}:=\frac{r_{j}}{s_{j}}-\frac{p_{j}}{q_{j}}
$$

By [36, Th. 6.1], in fact $\varepsilon_{j}=\frac{1}{q_{j} s_{j}}$, and so $\lim _{i \rightarrow \infty} \varepsilon_{i} b_{i}=0$.

In fact, one can calculate the energy $\overline{\mathcal{E}}$ of $\mathbf{u}$ when the rotation angle is $\frac{a}{b} \pi$ with $a$ even and $b$ odd, although Proposition 6 shows that, in this case, $\overline{\mathcal{E}}(\mathbf{u})$ does not correspond to any physical quantity. For example, based on the equality

$$
\max \{|a-b|+|b-c|+|c-a|: a, b, c \in[-1,1]\}=4,
$$

we can easily show that $\overline{\mathcal{E}}(\mathbf{u})=\frac{8}{3} \pi$ if the rotation angle $\theta_{0}$ in the definition (32) of $\mathbf{u}$ is $\frac{2}{3} \pi$.

The fact that $\mathcal{E}$ measures the area of the created surface will be shown in a forthcoming paper [30]. 


\section{Discussion}

In this final section we comment on other models for cavitation, with or without fracture, that have been previously proposed. We discuss, in particular, some models suggested by Müller and Spector [34], Giaquinta, Modica and Souček [29], and Mucci [31].

In what follows we use $G_{\mathbf{u}}$ to denote the current carried by the graph of $\mathbf{u}$, and $\mathbb{M}\left(\partial G_{\mathbf{u}}\right)$ to denote the mass of its boundary, as is customary in the theory of currents.

Apart from proving the existence of minimizers and analyzing their model of cavitation based on the minimization of (2), Müller and Spector mentioned [34, p. 6] that penalty terms more general that Per $\mathbf{u}(\Omega)$ could be considered, and proposed $\mathbb{M}\left(\partial G_{\mathbf{u}}\right)$ as an example. As they indicated, the existence of minimizers for the resulting model follows from the Federer-Fleming [20] closure theorem. They also pointed out [34, p. 54] that "the mass of the boundary of the current accounts for all newly created surface, whether or not the surface is in contact with other pieces of surface". This latter feature (that two pieces of created surface have been put together) is the main characteristic of their example [34, Sect. 11], which we have recalled in Section 3.

In the particular setting of Sobolev deformations, that observation of Müller and Spector corresponds to our derivation of the energy functional $\mathcal{E}$ as a correct expression for the area of the created surface; this was obtained in Section 3 for the case of deformations that are smooth except for the formation of cavities and closed cracks, and will be proved in [30] in its full generality. Indeed, as we mentioned in Section 4 , our energy $\mathcal{E}(\mathbf{u})$ coincides with the mass $\mathbb{M}\left(\left(\partial G_{\mathbf{u}}\right)_{(n-1)}\right)$ of the $n-1$ vertical part of the boundary of $G_{\mathbf{u}}$, and in the case of deformations $\mathbf{u}$ in $W^{1, n-1}$, the currents $\left(\partial G_{\mathbf{u}}\right)_{(n-1)}$ and $\partial G_{\mathbf{u}}$ coincide (see, e.g., [28, Rk. 3.2.3.3]). In the more general case of cavitation and fracture, however, our results differ. What we have obtained in this paper is that it is not necessary to control the mass of the whole boundary of $G_{\mathbf{u}}$, but, rather, that it is sufficient to control its $n-1$ vertical part in order to build an existence theory (see Theorems 2 and 3). Furthermore, in this more general setting, the quantity $\mathcal{E}(\mathbf{u})$ retains its interpretation as the area of the surface created by $\mathbf{u}$.

The idea that a term related to the mass of the boundary of $G_{\mathbf{u}}$ could be added to the elastic energy also appeared in the monograph by Giaquinta, Modica and Souček [28, Sect. 2.6]. They mentioned that one can add to the elastic energy any lower semicontinuous functional that is coercive with respect to the mass of the boundary of the current (such as $\mathbb{M}\left(\partial G_{\mathbf{u}}\right)$ itself, as suggested in [34]). Thus, this is a valid model for elastic energy with a generalized concept of 'fracture'. However, in their book this was mentioned only briefly, and the sense in which that functional could measure a 'fracture' was not made precise. They did not pursue this argument further, except for the analysis of radial cavitation.

Finally, we discuss the work of Mucci [31]. His model is based on the minimization of

$$
\int_{\Omega} W(\mathbf{x}, \nabla \mathbf{u}(\mathbf{x})) \mathrm{d} \mathbf{x}+\sum_{k=0}^{n-1} \mathcal{H}^{k}\left(S_{k}(\mathbf{u})\right)
$$

in the class of functions $\mathbf{u}$ that are approximately differentiable, $\nabla \mathbf{u}$ and its minors of all orders are in $L^{p}$, and the quantities $\|\mathbf{u}\|_{L^{\infty}}$ and $\mathbb{M}\left(\partial G_{\mathbf{u}}\right)$ satisfy an a priori bound. The set $S_{k}(\mathbf{u})$ is defined as the union of the supports of the singular part of the distributional minors of order $n-k$ (see, e.g., $[6,33,28]$ for the definition and properties of the distributional determinant and the distributional minors). 
In the above model, the set $S_{k}(\mathbf{u})$ is presented as a $k$-dimensional fracture set. This interpretation requires the previous result that $S_{k}(\mathbf{u})$ is countably $\mathcal{H}^{k}$-rectifiable. However, this latter result (which is one of the claims of [31, Prop. 4.9]) is not valid. Indeed, consider for example the deformation $\mathbf{u}:(-1,1)^{2} \rightarrow \mathbb{R}^{2}$ defined by

$$
\mathbf{u}(\mathbf{x}):= \begin{cases}\mathbf{x} & \text { if } \mathbf{x} \in(-1,0) \times(-1,1), \\ \mathbf{x}+(1,0) & \text { if } \mathbf{x} \in(0,1) \times(-1,1)\end{cases}
$$

We have that $\nabla \mathbf{u}=\mathbf{1}$ a.e., and it is easy to check that

$$
\begin{aligned}
& \left\langle\operatorname{Det} \nabla \mathbf{u}-(\operatorname{det} \nabla \mathbf{u}) \mathcal{L}^{2}, \phi\right\rangle=\int_{(-1,1)^{2}}\left[-\frac{1}{2} \mathbf{u} \cdot(\operatorname{cof} \nabla \mathbf{u}) D \phi-\phi \operatorname{det} \nabla \mathbf{u}\right] \mathrm{d} \mathbf{x} \\
& =-\frac{1}{2}\left[\int_{(-1,0) \times(-1,1)}+\int_{(0,1) \times(-1,1)}\right] \operatorname{Div}[\phi(\operatorname{adj} \nabla \mathbf{u}) \mathbf{u}] \mathrm{d} \mathbf{x}=\frac{1}{2} \int_{-1}^{1} \phi\left(0, x_{2}\right) \mathrm{d} x_{2}
\end{aligned}
$$

for every $\phi \in C_{c}^{\infty}\left((-1,1)^{2}\right)$. Therefore,

$$
\operatorname{Det} \nabla \mathbf{u}=(\operatorname{det} \nabla \mathbf{u}) \mathcal{L}^{2}+\frac{1}{2} \mathcal{H}^{1}\left\llcorner J_{\mathbf{u}},\right.
$$

where the jump set $J_{\mathbf{u}}$ of $\mathbf{u}$ is given by $\{0\} \times(-1,1)$. Consequently, in this example, the support $S_{0}(\mathbf{u})$ of the singular part of the distributional Jacobian determinant is $\{0\} \times(-1,1)$, so $S_{0}(\mathbf{u})$ does not correspond to cavities (i.e., to 0-dimensional fractures), and it is not countably $\mathcal{H}^{0}$-rectifiable.

In the case of Sobolev deformations satisfying some invertibility conditions, Müller and Spector [34, Th. 8.4] proved that the support of the singular part of the distributional Jacobian determinant is countably $\mathcal{H}^{0}$-rectifiable, and corresponds precisely to the cavities created by the deformation $\mathbf{u}$. However, the example above shows that this is not true for maps with fractures. This is because the set $S_{k}(\mathbf{u})$ does not only detect fracture surfaces of dimension $k$, but, in fact, all created surfaces of dimensions $k$ and above.

The proof in [31, Prop. 4.9] that $S_{k}(\mathbf{u})$ is countably $\mathcal{H}^{k}$-rectifiable rests upon [31, Prop. 4.2], where the result is obtained for the case $k=0$. The proof of [31, Prop. 4.2], in turn, is based on the isoperimetric inequality [31, Prop. 3.1], which is presented as a generalization of the isoperimetric inequality in the proof of [34, Th. 8.4].

The claim of [31, Prop. 3.1] is that for every $\mathbf{x}_{0} \in \Omega$ and a.e. $r>0$ such that $\bar{B}\left(\mathbf{x}_{0}, r\right) \subset \Omega$,

$$
\mid\left\langle\left(\partial G_{\mathbf{u}}\right)\left\llcorner\left(\bar{B}\left(\mathbf{x}_{0}, r\right) \times \mathbb{R}^{n}\right), \omega_{n}\right\rangle\right| \leq c_{n} \mathbb{M}\left(\left(\partial G_{\mathbf{u}}\right)_{(n-1)}\left\llcorner\left(\bar{B}\left(\mathbf{x}_{0}, r\right) \times \mathbb{R}^{n}\right)\right)^{\frac{n}{n-1}},\right.
$$

where $c_{n}$ is the isoperimetric constant, and $\omega_{n}$ is a differential form of compact support such that

$$
\omega_{n}(\mathbf{x}, \mathbf{y})=\frac{1}{n} \sum_{j=1}^{n}(-1)^{j-1} y^{j} \widehat{\mathrm{d} y^{j}}
$$

for $\mathbf{x} \in \bar{B}\left(\mathbf{x}_{0}, r\right)$ and $\mathbf{y} \in \bar{B}\left(\mathbf{0},\|\mathbf{u}\|_{\infty}\right)$ (we refer the reader to [31] for an exposition of the concepts and the notation employed). The term

$$
\mathbb{M}\left(\left(\partial G_{\mathbf{u}}\right)_{(n-1)}\left\llcorner\left(\bar{B}\left(\mathbf{x}_{0}, r\right) \times \mathbb{R}^{n}\right)\right)\right.
$$


which coincides with our $\mathcal{E}(\mathbf{u})$, corresponds to the area of the surface created by $\mathbf{u}$ in the ball $B\left(\mathbf{x}_{0}, r\right)$. On the other hand, for all $\mathbf{g} \in C_{c}^{\infty}\left(\mathbb{R}^{n}, \mathbb{R}^{n}\right)$ and a.e. $r>0$ small, it can be seen that

$$
\begin{aligned}
\left\langle( \partial G _ { \mathbf { u } } ) \left\llcorner\left(\bar{B}\left(\mathbf{x}_{0}, r\right)\right.\right.\right. & \left.\left.\times \mathbb{R}^{n}\right), \omega_{\mathbf{g}}\right\rangle=\int_{B\left(\mathbf{x}_{0}, r\right)} \operatorname{div} \mathbf{g}(\mathbf{u}(\mathbf{x})) \operatorname{det} \nabla \mathbf{u}(\mathbf{x}) \mathrm{d} \mathbf{x} \\
& -\int_{\partial B\left(\mathbf{x}_{0}, r\right)} \mathbf{g}(\mathbf{u}(\mathbf{x})) \cdot \operatorname{cof} \nabla \mathbf{u}(\mathbf{x}) \frac{\mathbf{x}-\mathbf{x}_{0}}{\left|\mathbf{x}-\mathbf{x}_{0}\right|} \mathrm{d} \mathcal{H}^{n-1}(\mathbf{x}),
\end{aligned}
$$

where $\omega_{\mathbf{g}}$ is the differential form

$$
\omega_{\mathbf{g}}(\mathbf{x}, \mathbf{y}):=\sum_{j=1}^{n}(-1)^{j-1} g^{j}(\mathbf{y}) \widehat{\mathrm{d} y^{j}}
$$

Therefore,

$$
\mid\left\langle\left(\partial G_{\mathbf{u}}\right)\left\llcorner\left(\bar{B}\left(\mathbf{x}_{0}, r\right) \times \mathbb{R}^{n}\right), \omega_{n}\right\rangle|=|\left(\operatorname{Det} \nabla \mathbf{u}-(\operatorname{det} \nabla \mathbf{u}) \mathcal{L}^{n}\right)\left(\bar{B}\left(\mathbf{x}_{0}, r\right)\right)\right|,
$$

where Det $\nabla \mathbf{u}$ denotes the distributional Jacobian determinant of $\mathbf{u}$. Mucci's isoperimetric inequality, then, states that the singular part of the distributional determinant is controlled by the area of the created surface raised to the power of $n /(n-1)$.

If $\mathbf{u}$ is a Sobolev map satisfying condition (INV) then (41) provides the total volume of the cavities generated in $B\left(\mathbf{x}_{0}, r\right)$, whereas the area of the created surface coincides with the area of the boundary of those cavities. In this case, therefore, inequality (38) makes sense (see [34, Lemma 8.1] and [14, Lemma 4.3]), as it is truly an isoperimetric inequality. In contrast, in the more general case of deformations having discontinuities along $(n-1)$-dimensional surfaces, inequality (38) is not valid. This is because we cannot define a notion analogous to that of a cavity created; in other words, the created surface does not necessarily correspond to the boundary of an enclosed volume. As Det $\nabla \mathbf{u}-(\operatorname{det} \nabla \mathbf{u}) \mathcal{L}^{n}$ cannot be interpreted any more as a measure giving the volume of the cavities, it cannot be dominated by the area of the created surface raised to some exponent. An explicit example is given by the function u defined in (36). Indeed, if $0<r<1$ then, thanks to (37) and (41), we have that

$$
\left|\left\langle\left(\partial G_{\mathbf{u}}\right) \mathbf{L}\left(\bar{B}(\mathbf{0}, r) \times \mathbb{R}^{2}\right), \omega_{2}\right\rangle\right|=r,
$$

while

$$
\mathbb{M}\left(\left(\partial G_{\mathbf{u}}\right)_{(1)}\left\llcorner\bar{B}(\mathbf{0}, r) \times \mathbb{R}^{2}\right)=4 r\right.
$$

so (38) is not true.

The proof of [31, Prop. 3.1] is based on the equality

$$
\left\langle\left(\partial G_{\mathbf{u}}\right)\left\llcorner\left(\bar{B}\left(\mathbf{x}_{0}, r\right) \times \mathbb{R}^{n}\right), \omega_{\mathbf{g}}\right\rangle=\left\langle\partial\left(G_{\mathbf{u}} \mathbf{L}\left(B\left(\mathbf{x}_{0}, r\right) \times \mathbb{R}^{n}\right)\right), \omega_{\mathbf{g}}\right\rangle,\right.
$$

where $\mathbf{g}$ is any given function in $C_{c}^{\infty}\left(\mathbb{R}^{n}, \mathbb{R}^{n}\right)$, and $\omega_{\mathbf{g}}$ is the differential form (40). That equality is incorrect, since it is inconsistent with equations (39) and

$$
\left\langle\partial\left(G_{\mathbf{u}}\left\llcorner\left(B\left(\mathbf{x}_{0}, r\right) \times \mathbb{R}^{n}\right)\right), \omega_{\mathbf{g}}\right\rangle=\int_{B\left(\mathbf{x}_{0}, r\right)} \operatorname{div} \mathbf{g}(\mathbf{u}(\mathbf{x})) \operatorname{det} \nabla \mathbf{u}(\mathbf{x}) \mathrm{d} \mathbf{x} .\right.
$$


Acknowledgements We are greatly indebted to John Ball for our discussions, for having introduced us to this problem, and for his constant encouragement and support. We have benefited from many discussions with Endre Süli, Jan Kristensen and Christoph Ortner. We also thank Javier Cilleruelo for pointing out the equivalence between ii) and iii) of Proposition 6. D.H. acknowledges financial support from the Catholic University of Chile, the Presidente de la República Scholarship of the Chilean Ministry of Planning, the Clarendon Fund of the Oxford University Press, and The Queen's College Graduate Scholarship; C.M.-C. from Project CGL2006-00524/BOS of the Spanish Ministry of Education and Science, and from the EPSRC research programme New Frontiers in the Mathematics of Solids (OxMOS).

\section{References}

1. L. Ambrosio, A compactness theorem for a new class of functions of bounded variation, Boll. Un. Mat. Ital. B (7), 3 (1989), pp. 857-881.

2. - Existence theory for a new class of variational problems, Arch. Rational Mech. Anal., 111 (1990), pp. 291-322.

3. L. Ambrosio, On the lower semicontinuity of quasiconvex integrals in $\operatorname{SBV}\left(\Omega, \mathbf{R}^{k}\right)$, Nonlinear Anal., 23 (1994), pp. 405-425.

4. L. Ambrosio AND A. BRAIDEs, Energies in $S B V$ and variational models in fracture mechanics, in Homogenization and applications to material sciences (Nice, 1995), Gakkōtosho, Tokyo, 1995, pp. 1-22.

5. L. Ambrosio, N. Fusco, and D. Pallara, Functions of bounded variation and free discontinuity problems, Oxford University Press, New York, 2000.

6. J. M. BALL, Convexity conditions and existence theorems in nonlinear elasticity, Arch. Rational Mech. Anal., 63 (1976/77), pp. 337-403.

7. Global invertibility of Sobolev functions and the interpenetration of matter, Proc. Roy. Soc. Edinburgh Sect. A, 88 (1981), pp. 315-328.

8. - Discontinuous equilibrium solutions and cavitation in nonlinear elasticity, Philos. Trans. Roy. Soc. London Ser. A, 306 (1982), pp. 557-611.

9. J. M. BAll, J. C. Currie, And P. J. Olver, Null Lagrangians, weak continuity, and variational problems of arbitrary order, J. Funct. Anal., 41 (1981), pp. 135-174.

10. J. M. BAll AND F. Murat, $W^{1, p}$-quasiconvexity and variational problems for multiple integrals, J. Funct. Anal., 58 (1984), pp. 225-253.

11. B. Bourdin, G. A. Francfort, And J.-J. Marigo, The variational approach to fracture, J. Elasticity, 91 (2008), pp. 5-148.

12. A. Chambolle, A. Giacomini, and M. Ponsiglione, Crack initiation in brittle materials, Arch. Rational Mech. Anal., 188 (2008), pp. 309-349.

13. P. G. CiARlet AND J. NEČAS, Injectivity and self-contact in nonlinear elasticity, Arch. Rational Mech. Anal., 97 (1987), pp. 171-188.

14. S. Conti And C. De Lellis, Some remarks on the theory of elasticity for compressible Neohookean materials, Ann. Sc. Norm. Super. Pisa Cl. Sci. (5), 2 (2003), pp. 521-549.

15. G. Dal Maso, G. A. Francfort, and R. Toader, Quasistatic crack growth in nonlinear elasticity, Arch. Rational Mech. Anal., 176 (2005), pp. 165-225.

16. G. Dal Maso And G. Lazzaroni, Quasistatic crack growth in finite elasticity with noninterpenetration, Ann. Inst. H. Poincaré Anal. Non Linéaire. In press.

17. G. DAl MAso AND R. TOADER, A model for the quasi-static growth of brittle fractures based on local minimization, Math. Models Methods Appl. Sci., 12 (2002), pp. 1773-1799.

18. G. DAl MAso AND R. TOADER, A model for the quasi-static growth of brittle fractures: existence and approximation results, Arch. Rational Mech. Anal., 162 (2002), pp. 101-135.

19. H. Federer, Geometric measure theory, Springer-Verlag, New York, 1969.

20. H. Federer And W. H. Fleming, Normal and integral currents, Ann. of Math. (2), 72 (1960), pp. 458-520.

21. G. A. FRANCFORT AND C. J. LARSEN, Existence and convergence for quasi-static evolution in brittle fracture, Comm. Pure Appl. Math., 56 (2003), pp. 1465-1500.

22. G. A. FrancFort AND J.-J. MARIGO, Revisiting brittle fracture as an energy minimization problem, J. Mech. Phys. Solids, 46 (1998), pp. 1319-1342.

23. N. Fusco, C. Leone, A. Verde, And R. MArch, A lower semi-continuity result for polyconvex functionals in $S B V$, Proc. Roy. Soc. Edinburgh Sect. A, 136 (2006), pp. 321336. 
24. A. Gent, Cavitation in rubber: a cautionary tale, Rubber Chem. Tech., 63 (1991), pp. G49-G53.

25. A. Gent And C. WAng, Fracture mechanics and cavitation in rubber-like solids, J. Mater. Sci., 26 (1991), pp. 3392-3395.

26. A. Giacomini And M. Ponsiglione, Non-interpenetration of matter for SBV deformations of hyperelastic brittle materials, Proc. Roy. Soc. Edinburgh Sect. A, 138 (2008), pp. 1019-1041.

27. M. Giaquinta, G. Modich, And J. SoučeK, Cartesian currents, weak diffeomorphisms and existence theorems in nonlinear elasticity, Arch. Rational Mech. Anal., 106 (1989), pp. 97-159.

28. - Cartesian currents in the calculus of variations. I, Springer-Verlag, Berlin, 1998.

29. - Cartesian currents in the calculus of variations. II, Springer-Verlag, Berlin, 1998.

30. D. Henao AND C. MorA-Corral, Fracture surfaces and the regularity of inverses for $B V$ deformations. Preprint available at www.bcamath.org/documentos/archivos/ publicaciones/HenaoMora2.pdf.

31. D. Mucci, Fractures and vector valued maps, Calc. Var. Partial Differential Equations, 22 (2005), pp. 391-420.

32. S. Müller, Weak continuity of determinants and nonlinear elasticity, C. R. Acad. Sci. Paris Sér. I Math., 307 (1988), pp. 501-506.

33. - Det $=$ det. A remark on the distributional determinant, C. R. Acad. Sci. Paris Sér. I Math., 311 (1990), pp. 13-17.

34. S. Müller AND S. J. Spector, An existence theory for nonlinear elasticity that allows for cavitation, Arch. Rational Mech. Anal., 131 (1995), pp. 1-66.

35. S. MÜller, Q. TANG, AND B. S. YAN, On a new class of elastic deformations not allowing for cavitation, Ann. Inst. H. Poincaré Anal. Non Linéaire, 11 (1994), pp. 217-243.

36. I. Niven And H. S. Zuckerman, An introduction to the theory of numbers, John Wiley, New York, 1960.

37. N. Petrinic, J. L. Curiel Sosa, C. R. Siviour, And B. C. F. Elliott, Improved predictive modelling of strain localisation and ductile fracture in a Ti-6Al-4V alloy subjected to impact loading, Journal de Physique IV, 134 (2006), pp. 147-155.

38. N. Petrinic, C. R. Siviour, J. L. Curiel Sosa, And B. C. F. Elliott, On competing volumetric and deviatoric damage mechanisms in simulation of ductile fracture in Ti64 alloy at high rates of strain, in Proceedings of Euromech EMMC-10 Conference "Multiphase and multi-component materials under dynamic loading", Kazimierz Dolny, 2007, pp. $467-478$

39. W. Rudin, Real and complex analysis, McGraw-Hill, New York, second ed., 1974.

40. J. Sivaloganathan And S. J. Spector, On the existence of minimizers with prescribed singular points in nonlinear elasticity, J. Elasticity, 59 (2000), pp. 83-113.

41. Q. TANG, Almost-everywhere injectivity in nonlinear elasticity, Proc. Roy. Soc. Edinburgh Sect. A, 109 (1988), pp. 79-95.

42. M. Williams and R. Schapery, Spherical flaw instability in hydrostatic tension, Int. J. Fract. Mech., 1 (1965), pp. 64-71.

43. W. P. Ziemer, Weakly differentiable functions, Springer-Verlag, New York, 1989. 